\title{
DECREASE IN THE BRIGHTNESS OF THE COSMIC X-RAY AND SOFT GAMMA-RAY BACKGROUND TOWARD CLUSTERS OF GALAXIES
}

\author{
S. A. Grebenev ${ }^{1 *}$ and R. A. Sunyaev ${ }^{1,2}$ \\ Received February 25, 2019; revised September 18, 2019; accepted October 23, 2019
}

\begin{abstract}
We show that Compton scattering by electrons of the hot intergalactic gas in galaxy clusters should lead to peculiar distortions of the cosmic background X-ray and soft gamma-ray radiation — an increase in its brightness at $h \nu \lesssim 60-100 \mathrm{keV}$ and a drop at higher energies. The background distortions are proportional to the cluster gas surface density, in contrast to the intensity of the thermal gas radiation proportional to the density squared, which allows the most important cluster parameters to be measured. The spectral shape of the background distortions and its dependence on the gas temperature, optical depth, and surface density distribution law have been studied using detailed Monte Carlo computations and confirmed by analytical estimations. In the cluster frame the maximum of the background decrease due to the recoil effect occurs at $h \nu \sim 500-600 \mathrm{keV}$. The photoionization of hydrogen- and helium-like iron and nickel ions leads to additional distortions in the background spectrum - a strong absorption line with the threshold at $h \nu \sim 9 \mathrm{keV}$ (and also to an absorption jump at $h \nu \gtrsim 2 \mathrm{keV}$ for cold clusters). The absorption of intrinsic thermal radiation from the cluster gas by these ions also leads to such lines. In nearby $(z \lesssim 1)$ clusters the line at $h \nu \gtrsim 2 \mathrm{keV}$ is noticeably enhanced by absorption in the colder $\left(\sim 10^{6} \mathrm{~K}\right)$ plasma of their peripheral $(\lesssim 3 \mathrm{Mpc}$ ) regions; moreover, the absorption line at $h \nu \sim 1.3 \mathrm{keV}$, which does not depend on the properties of the hot cluster gas, splits off from it. The redshift of distant clusters shifts the absorption lines in the background spectrum (at $\sim 2, \sim 9$, and $\sim 500 \mathrm{keV}$ ) to lower energies. Thus, in contrast to the microwave background radiation scattering effect, this effect depends on the cluster redshift $z$, but in a very peculiar way. When observing clusters at $z \gtrsim 1$, the effect allows one to determine how the X-ray background evolved and how it was "gathered" with $z$. To detect the effect, the accuracy of measurements should reach $\sim 0.1 \%$. We consider the most promising clusters for observing the effect and discuss the techniques whereby the influence of the thermal gas radiation hindering the detection of background distortions should be minimal.
\end{abstract}

\section{DOI: $10.1134 / \mathrm{S} 1063773719120016$}

Keywords: cosmic background radiation, galaxy clusters, hot and warm-hot intergalactic plasma, Compton scattering, recoil effect, Doppler effect, photoionization, bremsstrahlung and recombination radiation.

\section{INTRODUCTION}

In recent years the effect of a decrease in the brightness of the cosmic microwave background radiation toward 'galaxy clusters has turned from an elegant theoretical idea (Sunyaev and Zel'dovich 1970, 1972, 1980, 1981; 'Sunyaev 1980; Zel'dovich and Sunyaev 1982) into one of the most important tools for studies in the field of ob'servational cosmology and astrophysics of the early Universe. The energy redistribution of photons in the back'ground radiation spectrum after their Thomson scattering by electrons of the hot $\left(k T_{\mathrm{e}} \sim 2-15 \mathrm{keV}\right)$ intergalactic cluster gas underlies this effect. In this case, a deficit of photons is formed in the low-frequency part of the spectrum (at energies $h \nu \lesssim 3.83 k T_{\mathrm{r}}$, where $T_{\mathrm{r}} \simeq 2.7 \mathrm{~K}$ is the current temperature of the cosmic microwave background radiation), i.e., a "negative" source (a "hole" in the background) appears, while a bright "positive"

\footnotetext{
*e-mail: grebenev@iki.rssi.ru
}

source with an unusual spectrum appears in the high frequency part. This effect is unique in that its action is determined by the optical depth of the cluster gas for scattering by electrons along the line of sight $\tau_{\mathrm{T}}=\sigma_{\mathrm{T}} \int N_{\mathrm{e}}(l) \mathrm{d} l$, i.e., it is proportional to the gas density and not to the density squared, as the brightness of the intrinsic thermal radiation from the hot gas. Here, $\sigma_{\mathrm{T}}$ is the Thomson scattering cross section. Surprisingly, the amplitude of the effect does not decrease with cluster distance (redshift $z$ ); the spectral shape of the background distortions does not depend on $z$ either. Owing to these properties, the effect is widely used to determine the parameters of clusters and to effectively search for them. The effect is successfully observed with the specially constructed SPT (South Pole Telescope, Carlstrom et al. 2002; Williamson et al. 2011; Bleem et al. 2015) and ACT (Atacama Cosmology Telescope, Hasselfield et al. 2013) telescopes and a number of other telescopes (Birkinshaw 1999); the Planck satellite (Ade 
et al. (Planck Collaboration) 2014, 2015, 2016a) has made an enormous contribution to the investigation of the effect.

In this paper we consider a similar effect - the distortions arising due to scattering by electrons of the hot gas of clusters in the cosmic X-ray background. The existence of such an effect has already been mentioned in Sunyaev and Zel'dovich (1981), Zel'dovich and Sunyaev (1982), and Khatri and Sunyaev (2019). Based on simple nonrelativistic $\left(h \nu \ll m_{\mathrm{e}} c^{2}\right)$ estimates, these authors concluded that it is impossible to directly observe the effect against the intrinsic thermal X-ray background of the cluster gas. At the same time, they noted that it is potentially important to take into account this effect when considering the thermal balance of the gas. In this paper we performed relativistically accurate Monte Carlo computations of the effect and investigated and discussed the prospects for its observation in a harder ( $h \nu \gtrsim 60 \mathrm{keV}$ ) energy range.

The X-ray background (diffuse) radiation differs from the cosmic microwave background radiation by its origin (it is a superposition of the radiation spectra of a large number of AGNs - active galactic nuclei and quasars), but, at the same time, it is also characterized by a high degree of isotropy and homogeneity. In the standard ( $h \nu \lesssim 10 \mathrm{keV}$ ) X-ray range from 60 to $80 \%$ of the background radiation has already been resolved into separate (point) sources by telescopes with grazing-incidence mirrors (see, e.g., Hasinger et al. 1998; Miyaji et al. 2000; Giacconi et al. 2001); another $\sim 10 \%$ of the background has been explained by the thermal radiation of the gas in galaxy clusters. An extrapolation of the X-ray spectra for detected AGNs to the harder $(h \nu \gtrsim 10 \mathrm{keV})$ energy range, which takes into account the evolution of their number and the composition of their population with $z$, shows that here they should also make a dominant contribution to the background spectrum (Sazonov et al. 2008; Ueda et al. 2014; Miyaji et al. 2015). It follows from the simulations by Ueda et al. (2014) that the observed background spectrum is formed mainly at $z \sim 1$ or slightly farther, but its shape changes little even at higher $z$, while the intensity (in the rest frame) decreases slowly (in the range $z \sim 1.5-3$ the emissivity of AGNs remains almost constant, see Miyaji et al. 2000; Ueda et al. 2014). To a first approximation, up to $z \lesssim 2$ we may neglect these changes and assume that the X-ray background, along with the cosmic microwave one, is subject only to the ordinary cosmological expansion (see, e.g., Madau and Efstathiou 1999). The increase in the fraction of AGNs with high luminosities at $z \gtrsim 1$ is of greater importance. This should lead to an enhancement of the background fluctuations. Later we will discuss whether the appearance of such AGNs affects the detection of the effect under consideration.

The action of Compton scattering on cosmic microwave and X-ray background photons also differs: when the cosmic microwave background radiation is scattered, the electron temperature exceeds the photon energy and, therefore, the photons gain energy due to the Doppler effect; when the X-ray radiation is scattered, the photon energy exceeds (or at least is comparable to) the electron temperature and, accordingly, the photons, as will be shown below, on average, lose their energy due to the recoil effect.

\section{THE X-RAY BACKGROUND SPECTRUM}

The broadband spectrum of the hard X-ray $(h \nu>3$ $\mathrm{keV}$ ) cosmic background radiation was measured with the instruments of the HEAO-1 observatory (Gruber et al. 1999a). Although the absolute normalization of the spectrum was subsequently the subject of discussion (the INTEGRAL observations gave a value greater by $\sim 10 \%$, Churazov et al. 2007), the shape of the background spectrum was confirmed. We will use the following fit to the background spectrum (Gruber et al. 1999a):

$S_{0}(E) \simeq\left\{\begin{array}{l}7.877 E^{-0.29} e^{-E / 41.13}, \quad \text { at } E<60 \\ 0.0259(E / 60)^{-5.5}+ \\ 0.504(E / 60)^{-1.58}+, \\ 0.0288(E / 60)^{-1.05}\end{array} \quad\right.$ at $E>60$.

Here, $S_{0}(E)$ is the energy flux expressed in $\mathrm{keV}$ $\mathrm{cm}^{-2} \mathrm{~s}^{-1} \mathrm{keV}^{-1} \mathrm{sr}^{-1}$ and $E$ is the photon energy $h \nu$ in $\mathrm{keV}$. This fit agrees well with the gamma-ray background measurements at energies $1 \mathrm{MeV}-100 \mathrm{GeV}$ by the COMPTEL and EGRET telescopes of the CGRO observatory. The undistorted background spectrum corresponding to this fit is indicated in Fig. 19 by the thick solid (blue) line. The dotted line indicates the spectrum corresponding to the extension of the soft component in Eq. (1) to the hard range.

As we will see below, the distortions in this spectrum that arise when the background radiation interacts with electrons of the hot gas in a galaxy cluster are fairly small and do not exceed the current accuracy of our knowledge of the spectrum shape and parameters. In this regard the effect being discussed seems more difficult to measure than the distortion of the microwave background radiation whose spectrum has an almost ideal Planckian shape. However, if the accuracy of X-ray and gamma-ray measurements will increase to the required level in future, then the undistorted spectrum will be remeasured simultaneously with the distorted one, which will allow the deviations to be revealed. The estimates of the relative deviations of the background spectrum presented in this paper remain valid.

\section{MONTE CARLO COMPUTATIONS}

As the initial approximation we will assume the hot gas in a cluster to be distributed spherically symmetrically with uniform electron density $N_{\mathrm{e}}$ and temperature $k T_{\mathrm{e}}$ within its radius $R_{\mathrm{c}}$. The optical depth of such a gas cloud for scattering calculated along the line of sight 

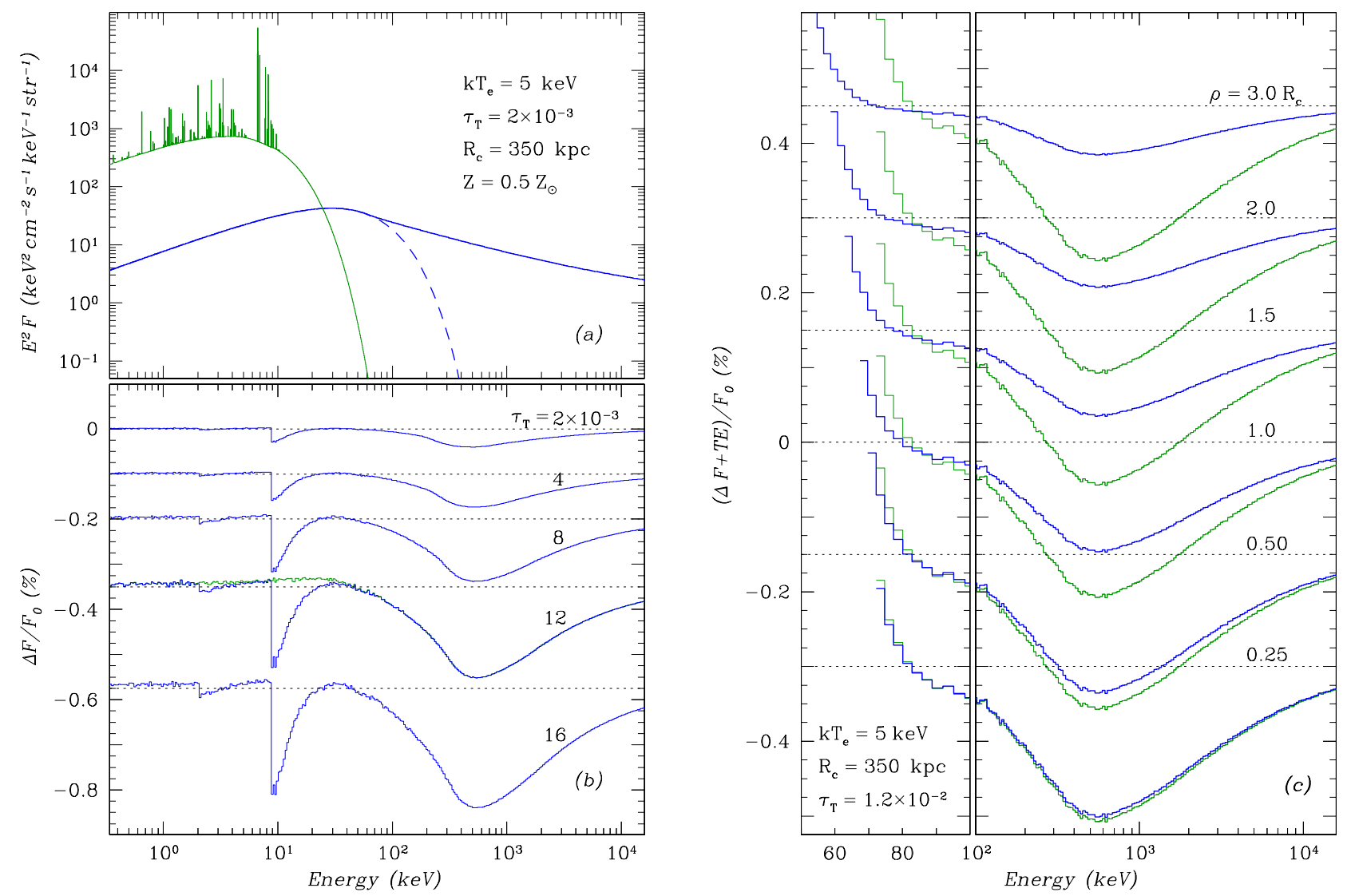

Fig. 1. (a) Spectra of the cosmic X-ray background (solid thick blue line) and the thermal (bremsstrahlung and recombination) X-ray radiation from the hot intergalactic gas (thin green line, 5 -eV resolution) of a cluster with a core radius $R_{\mathrm{c}}=350$ $\mathrm{kpc}$ and uniform electron temperature and density distributions inside it, $k T_{\mathrm{e}}=5 \mathrm{keV}$ and $N_{\mathrm{e}}=1.4 \times 10^{-3} \mathrm{~cm}^{-3}(\mathrm{so}$ that $\tau_{\mathrm{T}}=2 \times 10^{-3}$ ), the gas metallicity is $Z=0.5 Z_{\odot}$. The dashed line indicates the exponential extension of the background fit at low $(h \nu \lesssim 60 \mathrm{keV}$ ) energies. (b) The relative background distortions due to scattering by cluster gas electrons with the same temperature $k T_{\mathrm{e}}=5 \mathrm{keV}$, but different optical depths for Thomson scattering, $\tau_{\mathrm{T}}=2,4,8,12$ and $16 \times 10^{-3}$ (along the line of sight passing through the cluster center). (c) Attenuation of the distortions when they are observed at different impact parameters $\rho=0.25,0.5,1.0,1.5,2.0$ and $3.0 R_{\mathrm{c}}$ relative to the center for a cluster with a $\beta$ density distribution with $N_{\mathrm{c}}=7.5 \times 10^{-3} \mathrm{~cm}^{-3}$ and the same core radius and temperature as those on other panels. The thin (green) lines indicate the distortion profile at the center. The thermal gas radiation is included in the distortions. The dotted straight lines correspond to the unperturbed background for each case.

passing through its center will be $\tau_{\mathrm{T}}=2 \sigma_{\mathrm{T}} N_{\mathrm{e}} R_{\mathrm{c}}=2 \tau_{\mathrm{c}}$. Our computations of the Compton scattering of the background radiation in such a cloud were performed by the Monte Carlo method in accordance with the algorithms developed by Pozdnyakov et al. (1983). The background radiation was assumed to be incident on the cloud isotropically. The angle-averaged radiation leaving the cloud was considered as the emergent one. In this sense, characterizing below the amplitude of the distortions in the spectrum by the optical depth along the line of sight passing through the cloud center, by $\tau_{\mathrm{T}}$ we mean the characteristic of the cloud itself. The gas cloud with an optical depth $\tau_{\mathrm{T}}=1 \times 10^{-2}$ has a mass $M_{\mathrm{g}} \simeq 3.1 \times 10^{13}\left(\tau_{\mathrm{T}} / 0.01\right)\left(r_{\mathrm{c}} / 350 \mathrm{kpc}\right)^{2} M_{\odot}$. The total mass $M_{500}$ of the corresponding cluster, including the dark matter, should be greater at least by an order of magnitude. This is a moderate-mass cluster like the Coma cluster. Below we will also consider more massive clusters (see Table 4).

The hydrogen and helium in the cluster gas were assumed to have normal cosmic abundances, $X \simeq 0.74$ and $Y \simeq 0.24$ by mass (Allen et al. 1973), respectively, $N_{\mathrm{e}} \simeq(X+0.5 Y) \rho / m_{\mathrm{p}} \simeq 0.86 \rho / m_{\mathrm{p}}$. As a rule, the abundance of the iron-group elements was taken to be $Z=0.5 Z_{\odot}$, but it could change. At a gas temperature typical for clusters the atoms of most elements are ionized fully, iron is ionized to the hydrogen- and helium-like states, and nickel is ionized to the lithiumlike state. Photoabsorption by FeXXVI and FeXXV ions introduces distortions into the spectrum comparable in relative amplitude to the distortions due to scattering by electrons. Therefore, this process should be 
taken into account in the computations.

The degree of iron ionization for a plasma of the required temperature was obtained from the code by Raymond and Smith (1977), which is used to compute the ionization balance of an optically thin plasma. Apart from the Fe XXV and Fe XXVI ions, the absorption by FeXXII - FeXXIV ions was taken into account when computing the distortions of the background spectrum. The absorption by Ni XXIII - Ni XXVIII 1 was taken into account in the same way. The ionization by background photons in the ionization balance was neglected. We used the fits of the cross sections for photoabsorption by various ions from Verner and Yakovlev (1995) and Verner et al. (1996). The bremsstrahlung and recombination radiation spectrum of the intergalactic cluster plasma was also computed using the Raymond-Smith code. The thin solid (green) line in Fig.1 indicates this spectrum for a cluster with a plasma temperature $k T_{\mathrm{e}}=5 \mathrm{keV}$. On the whole, the recombination onto the iron atoms ionized as a result of cosmic background photoabsorption is analogous to the recombination onto the ions formed by collisional processes. The corresponding recombination radiation gives only a small $\lesssim 1 \%$ contribution to the intensity of the recombination radiation predicted by the Raymond-Smith code and does not change its spectrum.

\section{The Model with a Uniform Density Distribution}

Figure 1b presents the distortions in the background radiation spectrum (Eq. (1)) computed for various optical depths of the cloud2. The plasma temperature was assumed for all computations to be the same and equal to $k T_{\mathrm{e}}=5 \mathrm{keV}$, the heavy element abundance was $Z=0.5 Z_{\odot}$. It can be seen from the spectrum corresponding to $\tau_{\mathrm{T}}=1.2 \times 10^{-2}$, for which the thin (green) line indicates the result of our computation without absorption by iron ions, that Compton scattering slightly (by $\sim 0.02 \%$ ) raises the background intensity at energies $h \nu \lesssim 40 \mathrm{keV}$. However, photoabsorption reduces this rise beyond the iron ionization threshold $h \nu \gtrsim 9 \mathrm{keV}$ (blue line). In contrast, a dramatic drop in intensity reaching $\sim 0.2 \%$ near the threshold is observed in the background spectrum due to absorption at these energies. The maximal increase and decrease in intensity in the spectrum are $\lesssim 0.005$ and $\sim 0.03-0.04 \%$ for the spectrum corresponding to the smallest optical depth $\tau_{\mathrm{T}}=2 \times 10^{-3}$ of those considered and reach $\sim 0.03$ and $\sim 0.23 \%$, respectively, for the largest optical depth $\tau_{\mathrm{T}}=1.6 \times 10^{-2}$. The background spectrum also exhibits a weak absorption line on the $L$ shell of iron ions with the threshold at $h \nu \gtrsim 2 \mathrm{keV}$ and an amplitude of $\sim 0.04 \%$ (for a cluster

\footnotetext{
${ }^{1}$ In our computation of the photoabsorption in a warm-hot intergalactic medium with $k T_{\mathrm{e}}=0.2 \mathrm{keV}$ we took into account the Fe XIV - Fe XVII and Ni XIII - Ni XVII ions.

${ }^{2}$ Or various electron densities $N_{\mathrm{e}}$, because at a fixed cluster core radius the optical depth and density are related uniquely, $\tau_{\mathrm{T}} \sim N_{\mathrm{e}}$.
}

Table 1. Parameters of the $\mathrm{MeV}$ dip in the background spectrum toward a cluster with a uniform gas density ${ }^{\mathrm{a}}$

\begin{tabular}{c|c|c|c|c}
\hline \hline$\tau_{\mathrm{T}}$, & $h \nu_{\mathrm{th}}{ }^{\mathrm{b}}$ & $h \nu_{\gamma}{ }^{\mathrm{c}}$ & $h \Delta \nu_{\gamma}{ }^{\mathrm{d}}$ & $W_{\gamma}^{\mathrm{e}}$ \\
\hline$\times 10^{-3}$ & $\mathrm{keV}$ & $\mathrm{keV}$ & $\mathrm{MeV}$ & $\mathrm{keV}$ \\
\hline 2 & 29 & 584 & 2.3 & 1.1 \\
4 & 33 & 584 & 2.7 & 3.4 \\
8 & 35 & 584 & 2.9 & 10.0 \\
12 & 33 & 584 & 3.0 & 18.1 \\
16 & 35 & 604 & 3.0 & 27.1 \\
\hline
\end{tabular}

\footnotetext{
${ }^{\mathrm{a}} k T_{\mathrm{e}}=5 \mathrm{keV}$.

$\mathrm{b}$ The energy of the beginning of the dip.

c The energy of the deepest point of the dip.

$\mathrm{d}$ The FWHM of the dip.

e The equivalent width of the dip.
}

with $\tau_{\mathrm{T}}=1.6 \times 10^{-2}$ ).

At energies $h \nu \gtrsim 60 \mathrm{keV}$ scattering by electrons leads to a "dip" in the background spectrum due to the recoil effect. As a result of this effect, the photons lose a certain fraction of their energy and are shifted downward along the frequency axis. These shifted photons make a certain contribution to the intensity excess in the spectrum at energies $h \nu \lesssim 60 \mathrm{keV}$ (to be more precise, at $h \nu \lesssim k T_{\mathrm{e}}$, see the low-frequency asymptotics to Eq. (10) below and the curve corresponding to $k T_{\mathrm{e}}=0 \mathrm{keV}$ in Fig.(7). The soft photons shifted upward along the frequency axis (to $\left.h \nu \sim 3 k T_{\mathrm{e}}\right)$ due to the Doppler effect make a major contribution to the excess.

In the range $h \nu \sim 500-600 \mathrm{keV}$, where the depth of the dip in the background spectrum due to the recoil effect is maximal, the drop in background radiation brightness reaches $0.1-0.2 \%$ for realistic optical depths of the cluster, $\tau_{\mathrm{T}} \sim(4-12) \times 10^{-3}$. At $\tau_{\mathrm{T}}=1.6 \times 10^{-2}$ the dip deepens to $\sim 0.25 \%$.

In Table 1 the depth of the $\mathrm{MeV}$ dip is given in terms of the equivalent width $W_{\gamma}$ for various Thomson optical depths $\tau_{\mathrm{T}}$ of the gas. Given that the line is broad and the background intensity along its profile can change greatly, we defined the equivalent width as follows: $W_{\gamma}=\int_{\nu_{\text {th }}}^{\infty}\left(F_{0}-F_{\nu}\right) / F_{0} d h \nu$, where $F_{0}(\nu)$ is the photon spectrum of the background $\left[S_{0}(\nu)=h \nu F_{0}(\nu)\right]$. We considered the same model cluster with a uniform density, $R_{\mathrm{c}}=350 \mathrm{kpc}$, and $k T_{\mathrm{e}}=5 \mathrm{keV}$. The table also gives the energies of the beginning, $h \nu_{\text {th }}$, and the center, $h \nu_{\gamma}$, of the dip in the spectrum and its full width at half maximum (FWHM) $h \Delta \nu_{\gamma}$. Whereas the $h \nu_{\text {th }}$ and $h \nu_{\gamma}$ variations in Table1 can be attributed to the error of our calculation, the changes in $h \Delta \nu_{\gamma}$ and $W_{\gamma}$ are real, they reflect a drop in the dip amplitude as $\tau_{\mathrm{T}}$ decreases.

Note that the dips in the background spectrum related to the recoil effect and photoabsorption are formed in the radiation going through the cluster from its back side. To a first approximation, their depth is proportional to the mean optical depth of the cluster gas in this direc- 


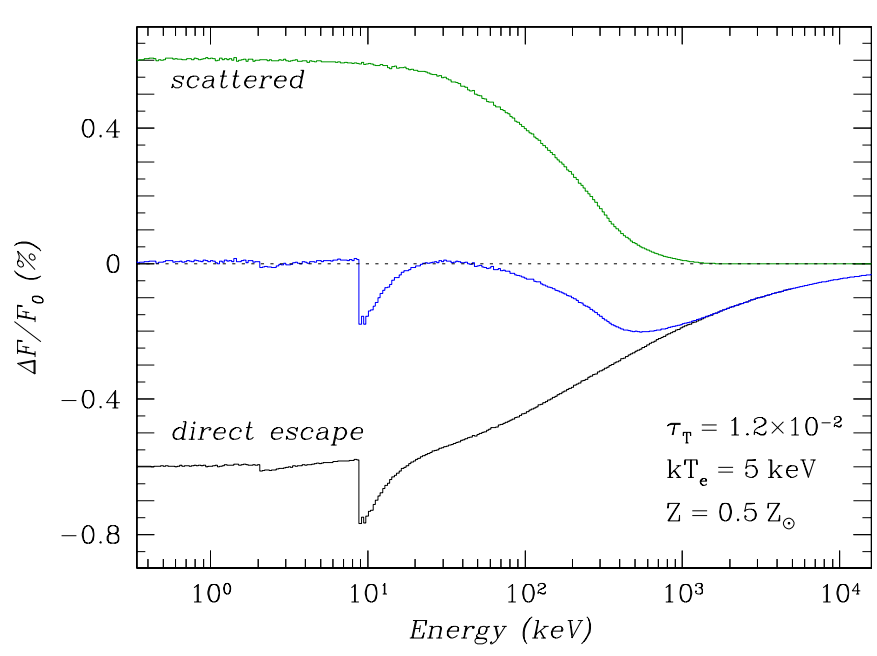

Fig. 2. Comparison of the background distortions arising in the hot cluster gas (relative to its initial spectrum) in the direct escape radiation (black curve) and scattered radiation (green curve). The blue (thick) curve indicates the sum of these distortions. The cluster gas is assumed to be distributed uniformly within the radius $R_{\mathrm{c}}=350 \mathrm{kpc}$ and to have an optical depth $\tau_{\mathrm{T}}=1.2 \times 10^{-2}$, temperature $k T_{\mathrm{e}}=5$ $\mathrm{keV}$, and metallicity $Z=0.5 Z_{\odot}$.

tion:

$$
<\tau_{\mathrm{T}}>=\frac{2}{R_{\mathrm{c}}^{2}} \int_{0}^{R_{\mathrm{c}}} \tau_{\mathrm{T}}(\rho) \rho d \rho=\frac{2}{3} \tau_{\mathrm{T}},
$$

where $\tau_{\mathrm{T}}(\rho)$ is the Thomson optical depth of the cluster along the line of sight passing at an impact parameter $\rho$ from the center. It is

$$
\tau_{\mathrm{T}}(\rho)= \begin{cases}\tau_{\mathrm{T}}\left(1-\rho^{2} / R_{\mathrm{c}}^{2}\right)^{1 / 2}, & \text { at } \rho<R_{\mathrm{c}} \\ 0, & \text { at } \rho \gtrsim R_{\mathrm{c}} .\end{cases}
$$

The amplitude of these dips in the background spectrum should be very sensitive to its spatial fluctuations. To reliably detect the effect of a decrease in background brightness at the corresponding energies, it is necessary to use extended (nearby) clusters.

In contrast, the increase in the background intensity below $h \nu<60 \mathrm{keV}$ is associated with the scattered photons. These are the photons of the radiation incident on the cluster from all sides, least of all from its back side (the photons coming from the back side are scattered at small angles and do not contribute noticeably to the spectral distortion). Figure2 2 shows separately the spectra of the distortions arising in the direct escape radiation and scattered radiation in a cluster with a temperature $k T_{\mathrm{e}}=5 \mathrm{keV}$ and a Thomson optical dept $\tau_{\mathrm{T}}=1.2 \times 10^{-2}$. It is clearly seen that all "negative" features (due to photoabsorption and the recoil effect) are contained only in the direct escape spectrum; the scattered photon spectrum is smooth and has no distinct features. Formally, the scattered photons are also subject to photoabsorption and are shifted to lower energies after recoil (in secondary interactions with the gas), but these effects are negligible due to the smallness of its optical depth.

The contribution of various sky regions to the scattered radiation spectrum is defined by the phase function, which in the nonrelativistic limit $h \nu \ll m_{\mathrm{e}} c^{2}$ has a simple form, $d \sigma_{\mathrm{T}}(\theta)=(3 / 8) \sigma_{\mathrm{T}}\left(1+\cos \theta^{2}\right) \sin \theta d \theta$, where $\theta$ is the scattering angle (and, in view of the symmetry of the phase function, the photon arrival angle relative to the line of sight toward the cluster). This is a smooth function. Clearly, the photons responsible for the increase in background intensity in the cluster due to the Doppler effect are collected from the entire sky and, hence, these distortions should not be sensitive to spatial background fluctuations.

\section{Dependence on the Gas Temperature}

Figure1 1 shows that the detection of distortions in the background spectrum in the X-ray range $h \nu \lesssim 60 \mathrm{keV}$ will be greatly complicated due to the intrinsic thermal radiation of the intergalactic gas.

The chances to detect the distortions increase for relaxed clusters with a lower temperature. This is illustrated by Fig. 3 in which the changes in the amplitude and shape of the distortion spectrum are shown as a function of the gas temperature. We again consider the model cluster with a uniform density distribution within the radius $R_{\mathrm{c}}=350 \mathrm{kpc}$ and an optical depth along the line of sight passing through the center $\tau_{\mathrm{T}}=1.2 \times 10^{-2}$. In Fig. [3 $\mathrm{k}$ the gas temperature in the cluster was assumed to be $2 \mathrm{keV}$. The figure gives a general idea of the relationship between the background spectrum and the thermal radiation spectrum of the cluster gas (just as Fig. [1], which gives such an idea for a cluster with $\left.k T_{\mathrm{e}}=5 \mathrm{keV}\right)$. Figure $3 \mathrm{~b}$ shows the relative distortions (in percent) arising in the background spectrum after scattering and absorption in the gas of such a cluster. We considered various gas temperatures $k T_{\mathrm{e}}=2,3,5,8,12,16$, and $20 \mathrm{keV}$.

The depth of the MeV dip in the background spectrum due to the recoil effect after scattering by electrons is almost independent of the gas temperature. As would be expected, the amplitude of other "negative" changes in the spectrum (the dips due to photoabsorption) reach its maximum for a cold gas with $k T_{\mathrm{e}}=2 \mathrm{keV}$. The depth of the dip beyond the threshold $h \nu \sim 9 \mathrm{keV}$ is $\sim$ $0.25 \%$. The amplitude of the line at $2 \mathrm{keV}$ also increases (to $\sim 0.1 \%$ ). These values refer to the case where the metallicity in the gas is $Z=0.5 Z_{\odot}$. The amplitude of the absorption lines changes as the metallicity increases and decreases. In the figure this change is indicated by the thin (green) lines for $k T_{\mathrm{e}}=2 \mathrm{keV}\left(Z=Z_{\odot}\right)$ and $k T_{\mathrm{e}}=20 \mathrm{keV}(Z=0)$.

In contrast to the "negative" changes in the background spectrum, the amplitude of the "positive" deviation (the excess of background radiation due to the 

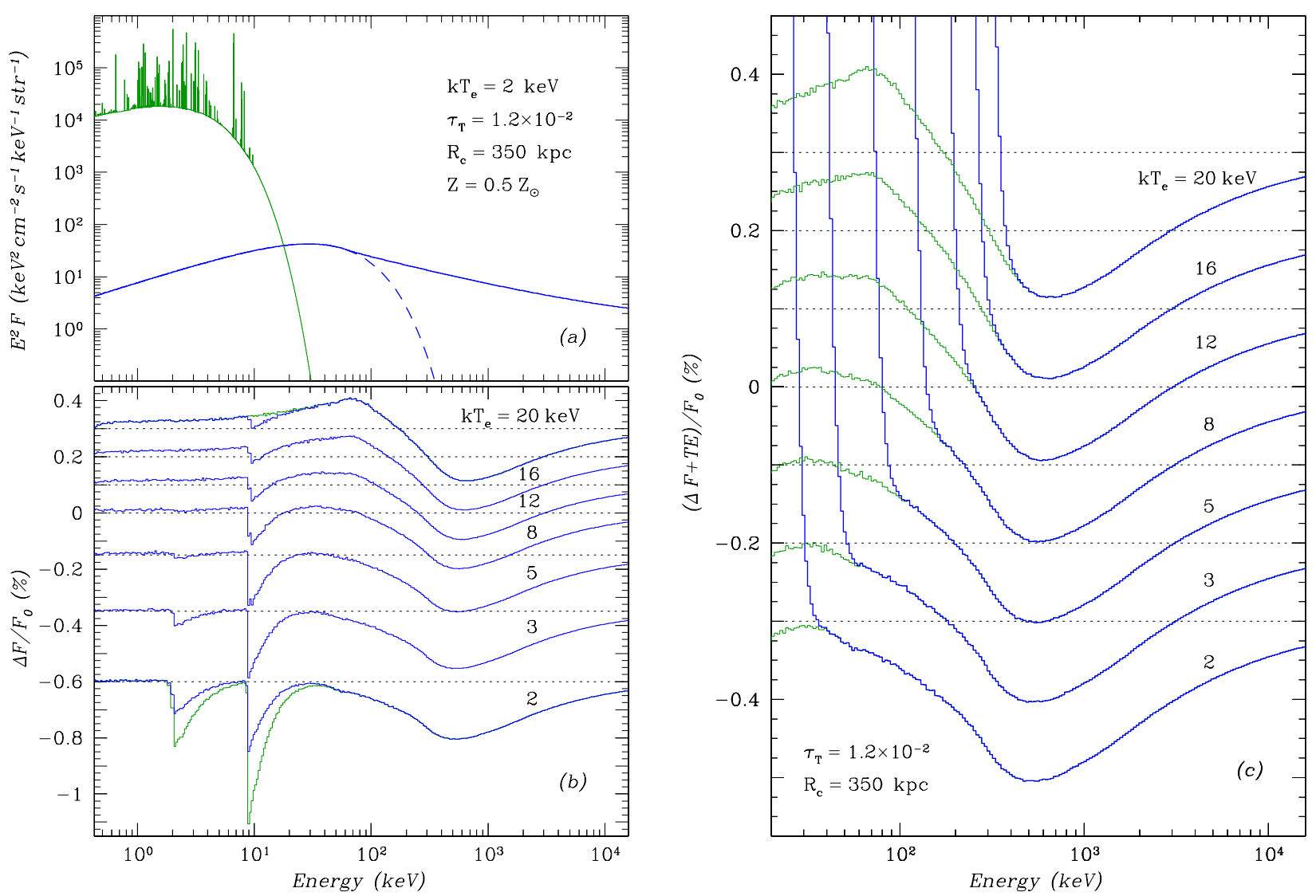

Fig. 3. Same as Fig. [1] but for $\tau_{\mathrm{T}}=1.2 \times 10^{-2}, R_{\mathrm{c}}=350 \mathrm{kpc}\left(N_{\mathrm{e}}=8.4 \times 10^{-3} \mathrm{~cm}^{-3}\right)$, and various temperatures: (a) $k T_{\mathrm{e}}=2$ $\mathrm{keV}$, (b, c) $k T_{\mathrm{e}}=2,3,5,8,12,16$, and $20 \mathrm{keV}$. The metallicity is $Z=0.5 Z_{\odot}$. The green line on panel (b) indicates our computation without photoabsorption $(Z=0)$ for $k T_{\mathrm{e}}=20 \mathrm{keV}$ and with photoabsorption at metallicity $Z=Z \odot$ for $k T_{\mathrm{e}}=2 \mathrm{keV}$. The background distortions on panel (c) include the thermal plasma radiation (the thin green lines — without this radiation).

Doppler effect) is maximal for the hottest gas with $k T_{\mathrm{e}}=20 \mathrm{keV}$. At energies $h \nu \sim 60-80 \mathrm{keV}$ a broad emission feature (line) whose relative amplitude reaches $\sim 0.1-0.15 \%$ is formed in the corresponding spectrum of the background distortions partly due to the Compton processes and partly due to the properties of the background spectrum itself.

In Fig. 3r the thermal radiation of the intergalactic gas was added to the background distortions (the measurements are assumed to be performed toward the cluster center). We see that the energy range that allows the Compton distortions to be directly observed in the background spectrum, without any illumination by the thermal gas radiation, turns out to be sufficiently wide only for relaxed clusters with a gas temperature $k T_{\mathrm{e}} \lesssim 5 \mathrm{keV}$. For young clusters (or clusters that have recently experienced a tidal effect from another close cluster), where the gas has a higher temperature, the lower boundary of the range admitting a direct observation of the effect is shifted into the gamma-ray energy range $h \nu \sim 400 \mathrm{keV}$.

Note that the iron and nickel ions were assumed to be at rest when computing the photoabsorption line pro- files. This is admissible, because the Doppler broadening and smearing related to the thermal motion of ions, for example, for the profile of the absorption jump at $h \nu_{\text {th }}=9 \mathrm{keV}$ at typical gas temperatures for clusters is only $\sim h \nu_{\text {th }}\left(2 k T_{\mathrm{e}} / 56 m_{\mathrm{p}} c^{2}\right)^{1 / 2} \sim 5\left(k T_{\mathrm{e}} / 5 \mathrm{keV}\right)^{1 / 2}$ $\mathrm{eV}$. The resolution of the computations presented in Figs. 1 and 3 and most of the succeeding figures, $h \Delta \nu \sim$ $0.0345 h \nu_{\text {th }} \simeq 300 \mathrm{eV}$, is much coarser. Even in Fig.4. which shows a detailed profile of this line (with a resolution that is better by several times), to demonstrate its complexity and multicomponent structure, the thermal motion of iron and nickel ions could smooth only slightly the sharpest features of the fine line structure.

Figure 4 shows that the edge of the absorption line near the threshold is strongly distorted even without any thermal broadening. It has the shape of a more or less regular "step" only in the case of $k T_{\mathrm{e}}=3 \mathrm{keV}$. At lower temperatures the beginning of both lines is shifted leftward, the threshold turns into a semblance of a "flight of stairs" consisting of several successive steps. At higher temperatures additional steps appear on the right, shifting the threshold of the lines by $300-400 \mathrm{eV}$ to greater 


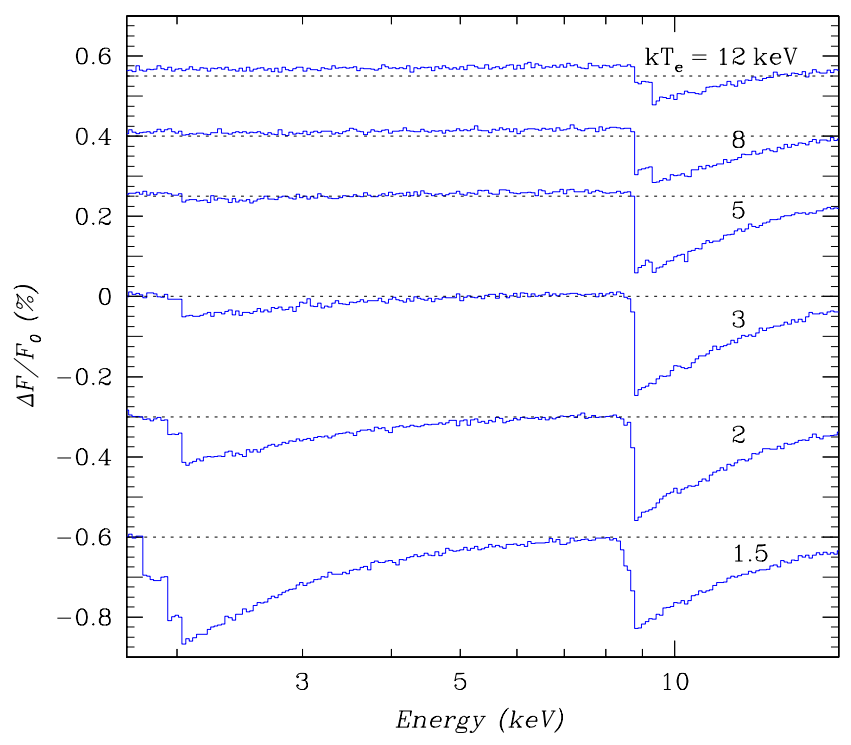

Fig. 4. Formation of absorption lines in the X-ray background spectrum due to photoionization of iron and nickel ions in the hot gas of a galaxy cluster. A cluster with a uniform density, optical depth $\tau_{\mathrm{T}}=1.2 \times 10^{-2}$, gas temperatures $k T_{\mathrm{e}}=1.5,2,3,5,8$, and $12 \mathrm{keV}$, and metallicity $Z=0.5 Z_{\odot}$.

energies. On reaching $k T_{\mathrm{e}}=8 \mathrm{keV}$, the absorption line with the threshold at $\sim 2 \mathrm{keV}$ virtually disappears, the amplitude of the hard line at $h \nu \sim 9 \mathrm{keV}$ also decreases.

Table 2 gives basic parameters of the line with the threshold at $\sim 9 \mathrm{keV}$, including its equivalent width $W_{\mathrm{X}}$. Since this line is formed in a region of enhanced background flux (through the Compton continuum forming in the cluster due to the Doppler effect after scattering by electrons), its equivalent width $W_{\mathrm{X}}=\int_{\nu_{\mathrm{th}}}^{\infty}\left(F_{\mathrm{c}}-\right.$ $\left.F_{\nu}\right) / F_{\mathrm{c}} d h \nu$ was determined relative to this enhanced level $F_{\mathrm{c}}(\nu)$ (the background photon spectrum distorted in the cluster obtained in the limit $Z \rightarrow 0$ ). The table presents the dependence of line parameters on the gas temperature and metallicity. As the temperature rises, the effective threshold of the line $h \nu_{\text {th }}$ and the energy of the deepest point of its profile $h \nu_{\mathrm{X}}$ are shifted by $400-500 \mathrm{eV}$ to higher energies. The cause can be understood from Fig. 4 - a new step related to the absorption of photons by more strongly ionized iron and nickel ions appears in the structure of the left line edge. For the same reason, the line width $h \Delta \nu_{\mathrm{X}}$ (FWHM ), which slightly increases with temperature probably due to a change in the shape of the Compton continuum, decreases abruptly by $\sim 500 \mathrm{eV}$ on reaching $k T_{\mathrm{e}}=12 \mathrm{keV}$. At this time the step height reaches half the absorption line depth.

\section{The Model with a $\beta$ Density Distribution}

Peripheral observations of the gas in clusters with a real (decreasing with radius) density distribution could also
Table 2. Parameters of the photoabsorption line with the threshold at $\sim 9 \mathrm{keV}$ in the $\mathrm{X}$-ray background spectrum toward a cluster with a uniform gas density ${ }^{\mathrm{a}}$

\begin{tabular}{c|c|c|c|c}
\hline \hline$k T_{\mathrm{e}}$, & $h \nu_{\mathrm{th}} \mathrm{b}$ & $h \nu_{\mathrm{x}}^{\mathrm{c}}$ & $h \Delta \nu_{\mathrm{X}}^{\mathrm{d}}$ & $W_{\mathrm{x}}^{\mathrm{e}}$ \\
\hline $\mathrm{keV}$ & $\mathrm{keV}$ & $\mathrm{keV}$ & $\mathrm{keV}$ & $\mathrm{eV}$ \\
\hline \multicolumn{5}{c}{$Z=0.5 Z_{\odot}$} \\
\hline 1.5 & $8.29^{\mathrm{f}}$ & $8.83^{\mathrm{f}}$ & 2.98 & 12.7 \\
2 & 8.38 & 8.83 & 3.06 & 14.0 \\
3 & 8.58 & 8.83 & 3.18 & 13.6 \\
5 & 8.58 & 8.83 & 3.69 & 11.9 \\
8 & 8.68 & 9.35 & 3.91 & 8.4 \\
12 & 8.68 & 9.35 & 3.43 & 5.3 \\
\hline \multicolumn{5}{|c}{$Z=1.0 Z_{\odot}$} \\
\hline \multirow{5}{*}{1.5} & 8.29 & 8.83 & 3.03 & 25.5 \\
2 & 8.38 & 8.83 & 3.08 & 28.1 \\
3 & 8.58 & 8.83 & 3.14 & 27.4 \\
5 & 8.58 & 9.35 & 3.70 & 23.9 \\
8 & 8.68 & 9.35 & 3.86 & 17.0 \\
12 & 8.68 & 9.35 & 3.38 & 10.7 \\
\hline
\end{tabular}

${ }^{\mathrm{a}} \tau_{\mathrm{T}}=1.2 \times 10^{-2}$.

$\mathrm{b}$ The threshold energy.

c The energy of the deepest point of the line.

d FWHM.

e The equivalent width.

$\mathrm{f}$ The value reflects the resolution of our computation.

have a certain advantage in combatting the thermal radiation. Indeed, the thermal radiation intensity is proportional to $N_{\mathrm{e}}^{2}$, while the distortions due to scattering by electrons are proportional to $N_{\mathrm{e}}$. Accordingly, the contribution of the thermal radiation should drop to the cluster edge faster than that of the scattered one (Zel'dovich and Sunyaev 1982).

The action of this effect is demonstrated by Fig.11, in which the distortions of the background spectrum (including the thermal plasma radiation) are represented by the thick (blue) lines for various impact parameters $\rho$ from the cluster center. For comparison, the thin (green) line indicates the spectrum of the distortions that should be observed toward the center. In the case of large $\rho$ the lines intersect, suggesting that the contribution of the thermal radiation vanishes at lower energies than those for the observations toward the cluster center. The spectra shown in this figure were computed for a $\beta$ gas density distribution (Cavaliere and FuscoFemiano 1976), which agrees satisfactorily with the observed X-ray brightness distribution of many clusters (Arnaud 2009),

$$
N_{\mathrm{e}}=N_{\mathrm{c}}\left(1+\frac{R^{2}}{R_{\mathrm{c}}^{2}}\right)^{-3 \beta / 2} .
$$

It follows from observations (Jones and Forman 1984) that for most clusters $\beta \simeq 2 / 3$. At such $\beta$ the cluster sur- 
face emission measure $E M(\rho)=2 \int_{0}^{\infty} N_{\mathrm{e}}^{2}(\rho) d l$, defining the thermal radiation intensity, and the Thomson optical depth along the line of sight $\tau_{\mathrm{T}}(\rho)=2 \sigma_{\mathrm{T}} \int_{0}^{\infty} N_{\mathrm{e}}(\rho) d l$, defining the amplitude of the spectral distortions due to scattering and absorption, at an impact parameter $\rho$ are, respectively,

$$
\operatorname{EM}(\rho)=\frac{\pi}{2}\left(1+\frac{\rho^{2}}{R_{\mathrm{c}}^{2}}\right)^{-3 / 2} N_{\mathrm{c}}^{2} R_{\mathrm{c}}
$$

and

$$
\tau_{\mathrm{T}}(\rho)=\pi\left(1+\frac{\rho^{2}}{R_{\mathrm{c}}^{2}}\right)^{-1 / 2} \sigma_{\mathrm{T}} N_{\mathrm{c}} R_{\mathrm{c}} .
$$

The gas emission measure $E M$ in a real cluster is seen to drop with increasing $\rho$ much faster than the optical depth $\tau_{\mathrm{T}}$. Therefore, at large $\rho$ the thermal radiation should cease to hinder the background observations at lower energies than in the observations toward the cluster center. This is illustrated by Fig.11, based on Eqs. (5) and (6). Unfortunately, the extension of the range favorable for the observation of background distortions after scattering turns out to be moderately large, while the amplitude of the effect proper, in turn, decreases quite rapidly with increasing $\rho$.

In the computation whose result is used here, as before, the intergalactic gas temperature was taken to be $k T_{\mathrm{e}}=5 \mathrm{keV}$, the cluster core radius is $R_{\mathrm{c}}=350$ $\mathrm{kpc}$, and the Thomson optical depth of the gas along the line of sight passing through the cluster center is $\tau_{\mathrm{T}}=1.2 \times 10^{-2}$. The distortions in the background spectrum toward the center (at $\rho=0$ ) were computed by the Monte Carlo method by assuming the density profile to break at the "outer" radius $R_{\mathrm{b}}=2 R_{\mathrm{c}}$.

The dependence of the results of our computations on $R_{\mathrm{b}}$ is investigated in Fig. The spectra of the background distortions arising in clusters with a $\beta$ gas density distribution, the same temperatures $k T_{\mathrm{e}}$ and optical depths along the line of sight toward the center $\tau_{\mathrm{T}}$, but different break radii of the $\beta$ density profile $R_{\mathrm{b}}=1,2,5$, and $10 R_{\mathrm{c}}$ are shown here. For comparison, the thin lines indicate our computations of the background distortions in a cluster with a uniform density distribution (with the same $k T_{\mathrm{e}}, R_{\mathrm{c}}$, and $\tau_{\mathrm{T}}$ ).

A change in $R_{\mathrm{b}}$ slightly changes the optical depth of the gas $\tau_{\mathrm{T}}\left(\rho=0, R_{\mathrm{b}}\right)$ in a real cluster relative to $\tau_{\mathrm{T}}(\rho=0)=\pi \sigma_{\mathrm{T}} N_{\mathrm{c}} R_{\mathrm{c}}$ following from Eq. (6) (derived in the limit $\left.R_{\mathrm{b}} \rightarrow \infty\right)$. Nevertheless, it is appropriate to compare the clusters of equal optical depths for the pure effect of different cluster geometries to be seen. For this purpose, in the computations in Fig. 5 the central density $N_{\mathrm{c}}$ of the cluster profile in Eq. (4) was multiplied by $0.5 \pi / \arctan \left(R_{\mathrm{b}} / R_{\mathrm{c}}\right)$. With or without this correction, the model clusters considered, of course, cannot be deemed identical, if only because the cluster gas mass increases noticeably with $R_{\mathrm{b}}$ from $M_{\mathrm{g}}=3.0 \times 10^{13} M_{\odot}$ for $R_{\mathrm{b}}=1 R_{\mathrm{c}}$ to $M_{\mathrm{g}}=6.4 \times 10^{14} M_{\odot}$ for $R_{\mathrm{b}}=10 R_{\mathrm{c}}$.

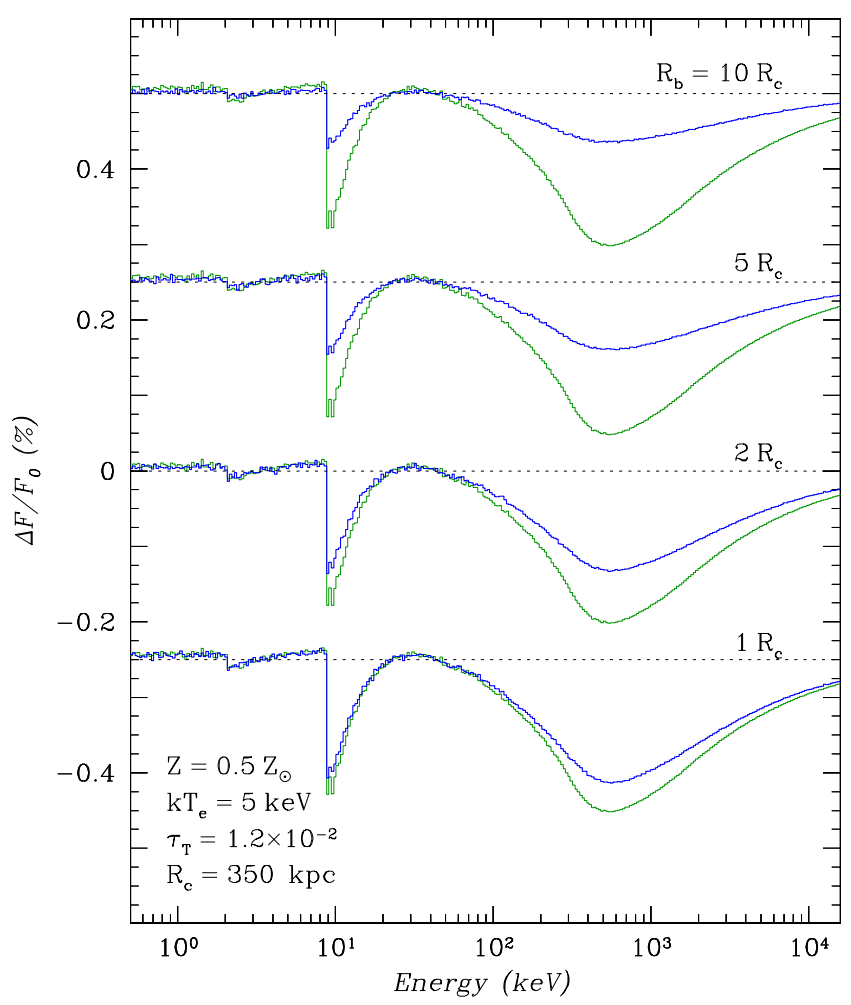

Fig. 5. Comparison of the relative background distortions arising in a "real" galaxy cluster (thick blue lines) with a $\beta$ gas density distribution (Eq. (4) ) and a cluster with a uniform density distribution (thin green line). The gas in the clusters has the same Thomson optical depths $\tau_{\mathrm{T}}=1.2 \times 10^{-2}$ (along the line of sight through the cluster center), core radii $R_{\mathrm{c}}=350 \mathrm{kpc}$, electron temperatures $k T_{\mathrm{e}}=5 \mathrm{keV}$, and $Z=$ $0.5 Z_{\odot}$. We considered the cases with various break radii $R_{\mathrm{b}}$ of the $\beta$ profile.

Figure 5 shows that the cluster with a real density distribution with $R_{\mathrm{b}}=1 R_{\mathrm{c}}$ leads to virtually the same background distortions in amplitude and shape of the energy dependence as does the cluster with a uniform density distribution. It can also be seen from the figure that even despite the increase in the mass of the cluster with a real density distribution with increasing $R_{\mathrm{b}}$, the amplitude of the background distortions decreases rapidly in this case. This behavior can be explained by taking into account the fact that the Thomson optical depth of the intergalactic gas averaged over the visible area of the cluster with a $\beta$ profile, $\left\langle\tau_{\mathrm{T}}\right\rangle$, decreases with increasing $R_{\mathrm{b}}$. Indeed, by integrating the optical depth from Eq. (6) over the area $2 \pi \int_{0}^{R_{\mathrm{b}}} \tau_{\mathrm{T}}(\rho) \rho d \rho$ and normalizing to $\pi R_{\mathrm{b}}^{2}$, we find

$$
<\tau_{\mathrm{T}}\left(R_{\mathrm{b}}\right)>=2 \tau_{\mathrm{T}} \frac{R_{\mathrm{c}}^{2}}{R_{\mathrm{b}}^{2}}\left[\left(1+\frac{R_{\mathrm{b}}^{2}}{R_{\mathrm{c}}^{2}}\right)^{1 / 2}-1\right]
$$

$\simeq 0.83 \tau_{\mathrm{T}}$ at $R_{\mathrm{b}}=1 R_{\mathrm{c}}$ and $\simeq 0.18 \tau_{\mathrm{T}}$ at $R_{\mathrm{b}}=10 R_{\mathrm{c}}$. 


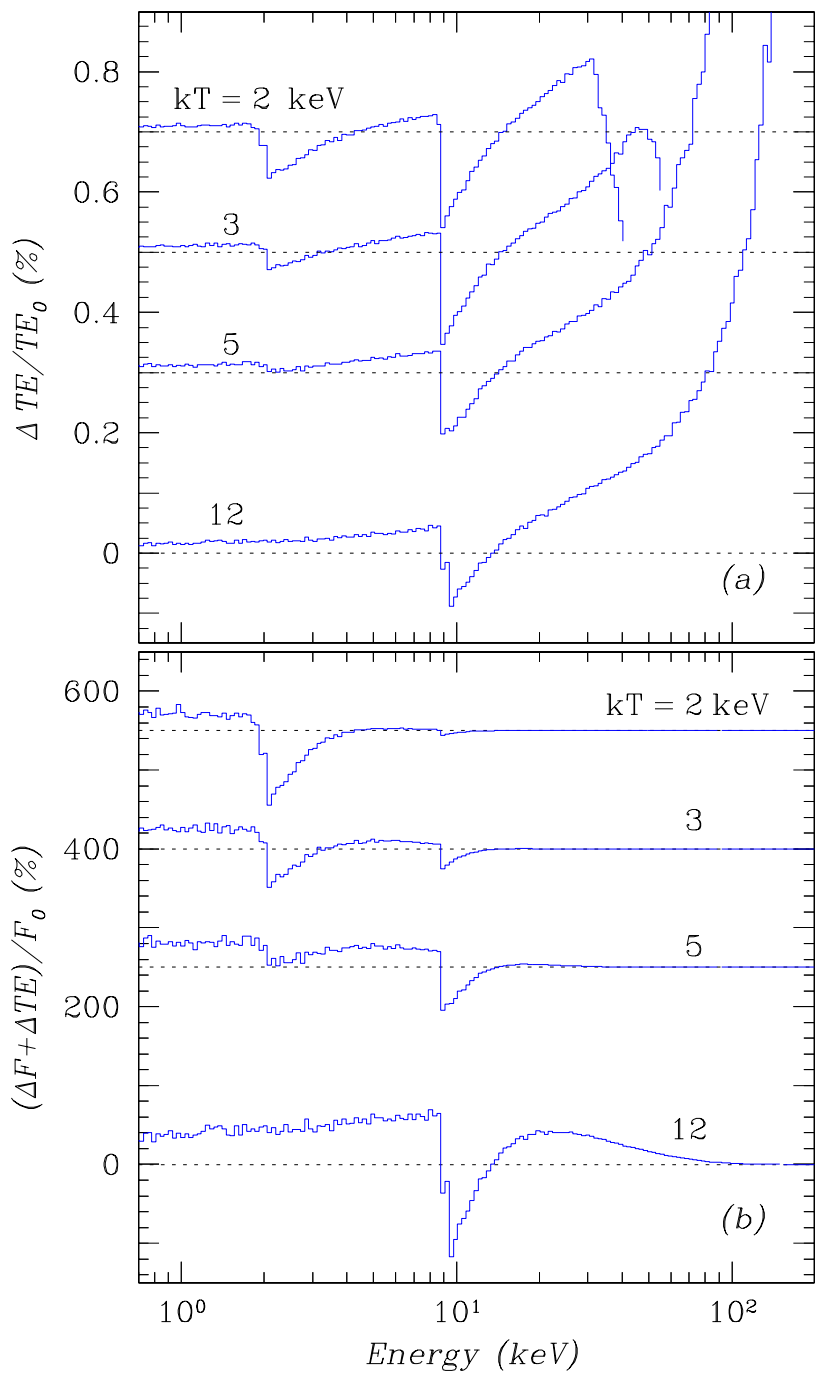

Fig. 6. Formation of absorption lines in the thermal radiation spectrum of the cluster gas due to the ionization of iron ions: relative to (a) the thermal radiation itself and (b) the cosmic background radiation. A cluster with a uniform density, radius $R_{\mathrm{c}}=350 \mathrm{kpc}, \tau_{\mathrm{T}}=1.2 \times 10^{-2}, Z=0.5 Z_{\odot}$, and various gas temperatures $k T_{\mathrm{e}}$.

Note that we compute the average spectrum of the background distortions in the cluster by the Monte Carlo method. To obtain the background distortions toward its center $(\rho=0)$ presented in Fig. 1; , their amplitude was properly corrected for the above decrease in optical depth when averaged over the visible cluster area $\pi\left(2 R_{\mathrm{c}}\right)^{2}$.

¿From the viewpoint of effective detection of the background distortions due to the interaction with the cluster gas, Fig. 5 clearly indicates that the cluster observations by a telescope with an aperture (angular resolution) covering the central part of the cluster with a radius $\lesssim 2 R_{\mathrm{c}}$. A similar conclusion can be drawn from an analysis of the background distortions arising in a cluster with the density distribution predicted by the
Navarro-Frenk-White model (hereafter NFW, Navarro et al. 1997). Such a cluster is analyzed in the Appendix.

\section{Spectral Distortions of the Intrinsic Gas Radiation}

The detection of the background distortions associated with its interaction with the hot gas of galaxy clusters is complicated not just by the presence of intense intrinsic gas radiation. In turn, distortions whose amplitude exceeds noticeably the relative amplitude of the background distortions appear in the spectrum of this thermal radiation.

Indeed, the Raymond-Smith code, along with other codes used to compute the bremsstrahlung and recombination radiation spectrum of an optically thin plasma, suggests that the optical depth of the plasma $\tau_{\mathrm{T}} \rightarrow 0$ and, therefore, takes into account only the collisional processes and disregards the ionization of the iron-group elements by the intrinsic plasma radiation. It also disregards the Compton scattering of the plasma radiation inside the cluster. Meanwhile, as the results of our computations presented in this paper show, the optical depth of the cluster gas is enough for strong absorption lines at $h \nu \gtrsim 2$ and $9 \mathrm{keV}$ and other features to be formed in the spectrum when the background radiation passes through it. Obviously, such spectral distortions should also appear in the intrinsic gas radiation.

Figure 6a shows the results of our Monte Carlo computations of such distortions (in \% to the thermal radiation of the intergalactic gas) for a cluster with a uniform density with $\tau_{\mathrm{T}}=1.2 \times 10^{-2}, k T_{\mathrm{e}}=2,3,5$, or $12 \mathrm{keV}$ and $Z=0.5 Z_{\odot}$. We used the same code as that for the background distortion computations, but the source of photons was assumed to be uniformly distributed throughout the cluster and its radiation spectrum was taken from our computations of the optically thin plasma spectrum by the Raymond-Smith code for a cluster temperature $k T_{\mathrm{e}}$ (only the continuum bremsstrahlung was taken into account).

We see that the distortions arising in the spectrum of the thermal gas radiation resemble those in the background spectrum. However, there is also a difference. The bremsstrahlung spectrum abruptly breaks at energies $h \nu \gtrsim k T_{\mathrm{e}}$. At the same time, Comptonization shifts the photons upward along the frequency axis, tending to form a Wien spectrum with a characteristic break energy $\sim 3 k T_{\mathrm{e}}$. This process explains the sharp increase in radiation intensity at energies $h \nu \gtrsim k T_{\mathrm{e}}$. The process is well known in X-ray astronomy and is successfully used to explain the observed spectra of accreting black holes (see, e.g., Shapiro et al. 1976; Sunyaev and Titarchuk 1980). The upward shift of the photons along the frequency axis is determined by the Doppler effect; after each scattering the average change in photon frequency is $\Delta \nu / \nu \sim\left(k T_{\mathrm{e}} / m_{\mathrm{e}} c^{2}\right)^{1 / 2}$. A competing process is the recoil effect, which in the nonrelativistic limit lowers the photon frequency after scattering, on average, by $\Delta \nu / \nu \sim-h \nu / m_{\mathrm{e}} c^{2}$ (these estimates can be easily ob- 
Table 3. Parameters of the total (including the thermal radiation distortions) photoabsorption line with the threshold at $\sim 9 \mathrm{keV}$ in the X-ray background spectrum toward a cluster with a uniform gas density ${ }^{\mathrm{a}}$

\begin{tabular}{c|c|c|c|c}
\hline \hline$k T_{\mathrm{e}}$, & $h \nu_{\mathrm{th}}{ }^{\mathrm{b}}$ & $h \nu_{\mathrm{X}}{ }^{\mathrm{c}}$ & $h \Delta \nu_{\mathrm{X}}{ }^{\mathrm{d}}$ & $W_{\mathrm{X}}{ }^{\mathrm{e}}$ \\
\hline $\mathrm{keV}$ & $\mathrm{keV}$ & $\mathrm{keV}$ & $\mathrm{keV}$ & $\mathrm{keV}$ \\
\hline \multicolumn{5}{|c}{$Z=0.5 Z_{\odot}$} \\
\hline 2 & 8.19 & 8.93 & 1.13 & 0.11 \\
3 & 8.48 & 8.93 & 1.33 & 0.61 \\
5 & 8.48 & 8.93 & 2.16 & 2.22 \\
8 & 8.48 & 9.57 & 2.89 & 3.59 \\
12 & 8.48 & 9.57 & 2.77 & 3.72 \\
\hline \multicolumn{5}{|c}{$Z=1.0 Z_{\odot}$} \\
\hline 2 & 8.19 & 8.93 & 1.04 & 0.24 \\
3 & 8.48 & 8.93 & 1.36 & 1.24 \\
5 & 8.48 & 8.93 & 2.13 & 4.38 \\
8 & 8.48 & 9.57 & 2.93 & 7.34 \\
12 & 8.48 & 9.57 & 2.63 & 7.14 \\
\hline
\end{tabular}

${ }^{\mathrm{a}} R_{\mathrm{c}}=350 \mathrm{kpc}, \tau_{\mathrm{T}}=1.2 \times 10^{-2}$.

$\mathrm{b}$ The threshold energy.

c The energy of the deepest point of the line.

d FWHM.

$\mathrm{e}$ The equivalent width.

tained from the Kompaneets equation, see below). The processes are balanced at

$$
h \nu_{*} \sim\left(k T_{\mathrm{e}} m_{\mathrm{e}} c^{2}\right)^{1 / 2} \sim 32\left(k T_{\mathrm{e}} / 2 \mathrm{keV}\right)^{1 / 2} \mathrm{keV} .
$$

Accordingly, for clusters with a low gas temperature $k T_{\mathrm{e}} \lesssim 2-3 \mathrm{keV}$ a broad emission feature centered at energies $\sim 30-50 \mathrm{keV}$ is formed in the distortion spectrum (Fig.6); for hotter clusters the distortions grow with energy until the complete cutoff of the thermal spectrum. Formula (8) to some extent also explains the energy of the broad emission feature appearing in the distortion spectrum of the background in hot galaxy clusters (Fig. 3b).

The amplitude of the distortions in the thermal plasma radiation spectrum is $\sim \tau_{\mathrm{T}}$ and accounts for fractions of percent of the intensity of the spectrum itself (just as the amplitude of the cosmic background distortions). However, since the thermal radiation intensity in the X-ray range exceeds the background intensity by two or three orders of magnitude, these distortions are comparable to the background intensity. When the observed cluster radiation spectrum is fitted by the thermal radiation model of an optically thin plasma, these distortions, including the iron ion absorption lines, will not be subtracted and will lead to a noticeable enhancement of the distortions formed directly in the background spectrum. This is clearly seen from Fig.6b, in which the background distortions and the thermal gas radiation distortions are added together and are given in percent relative to the background spectrum. The background level is shown by dotted lines. The amplitude of the distortions in the iron and nickel photoabsorption lines reaches $100 \%$ or more. Note that the absorption line with the $9-\mathrm{keV}$ threshold greatly weakens in the distortion spectra of cold clusters with $k T_{\mathrm{e}}=2-3 \mathrm{keV}$ in Fig.6b, although in Fig.6a it has the largest amplitude among all of the clusters considered relative to the thermal radiation spectrum. This is due to the general rapid (exponential) drop in thermal radiation at these energies from cold clusters. The line with the threshold at $\sim 2$ $\mathrm{keV}$ in them still reaches a maximum. Table 3 gives the equivalent widths of the effective (total) absorption line at $9 \mathrm{keV}$ in the background spectrum in clusters with various gas temperatures and various iron abundances. Clearly, the intrinsic gas radiation distortions are very large and at energies $h \nu \lesssim 100 \mathrm{keV}$ exceed noticeably the background distortions. They are very difficult to take into account.

Below we will assume that the question about the subtraction of the thermal gas radiation spectrum, given its distortions due to the finite optical depth, from the observed cluster spectrum has somehow been solved. We will consider only the distortions arising directly in the cosmic background. The distortions of the intrinsic thermal gas radiation will be considered in detail in a separate paper (Grebenev and Sunyaev 2020).

\section{ANALYTICAL ESTIMATES}

When investigating the Compton scattering of the cosmic microwave background radiation in the hot gas of a galaxy cluster, important analytical estimates (Sunyaev and Zel'dovich 1980; Zel'dovich and Sunyaev 1982) were obtained by solving the Kompaneets (1957) equation, which describes the photon energy redistribution energy in the diffusion approximation. The validity of applying this equation to an optically thin gas typical for clusters was tested and confirmed by Sunyaev (1980). Similar quite interesting estimates can also be obtained for the problem under consideration.

The Kompaneets equation with relativistic corrections (Cooper 1971; see also Arons 1971; Illarionov and Sunyaev 1972; Grebenev and Sunyaev 1987), but at a moderately high electron temperature $\left(k T_{\mathrm{e}} \ll m_{\mathrm{e}} c^{2}\right)$ can be represented as

$$
\frac{\partial F_{\nu}}{\partial \tau_{\mathrm{c}}}=\frac{h}{m_{\mathrm{e}} c^{2}} \frac{\partial}{\partial \nu}\left[\frac{\nu^{4} \xi\left(T_{\mathrm{e}}\right)}{1+\beta \nu+\gamma \nu^{2}}\left(\frac{F_{\nu}}{\nu^{2}}+\frac{k T_{\mathrm{e}}}{h} \frac{\partial}{\partial \nu} \frac{F_{\nu}}{\nu^{2}}\right)\right],
$$

where $F_{\nu}$ is the intensity of the photon spectrum, $\tau_{\mathrm{c}}$ is the Thomson radial optical depth of the cloud, $\beta=$ $9 \times 10^{-3} \mathrm{keV}^{-1}, \gamma=4.2 \times 10^{-6} \mathrm{keV}^{-2}$,

$$
\xi\left(T_{\mathrm{e}}\right)=1+\frac{5}{2} \frac{k T_{\mathrm{e}}}{m_{\mathrm{e}} c^{2}} .
$$

We neglected the term responsible for the induced scattering and retained only the term of the first order in $k T_{\mathrm{e}} / m_{\mathrm{e}} c^{2}$ in $\xi\left(T_{\mathrm{e}}\right)$ (see Cooper 1971). Substituting the 


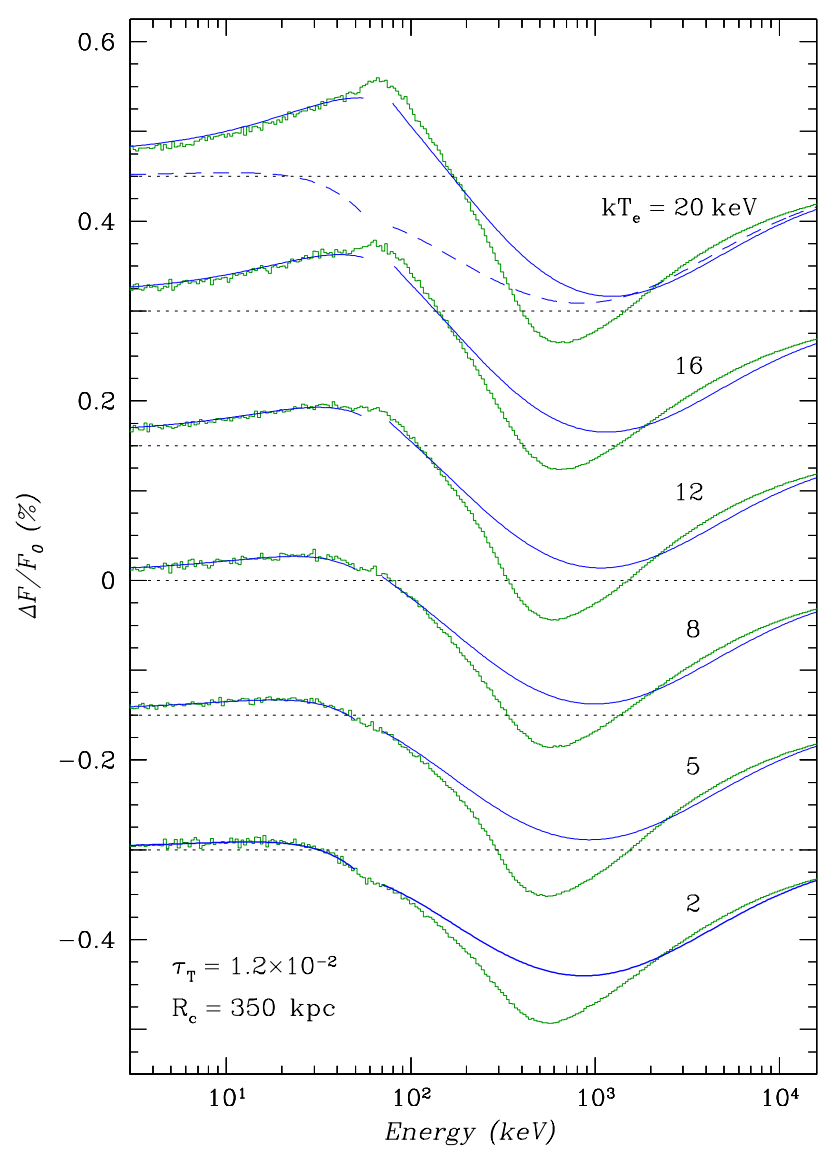

Fig. 7. Comparison of the relative hard X-ray background distortions due to scattering in the hot cluster gas calculated from the analytical formulas (9) and (10) (solid thick blue lines) and by the Monte Carlo method (thin green lines). The photoabsorption by iron ions was disregarded. We considered a cluster with a uniform density distribution, core radius $R_{\mathrm{c}}=350 \mathrm{kpc}$, optical depth $\tau_{\mathrm{T}}=1.2 \times 10^{-2}$, and various electron temperatures $k T_{\mathrm{e}}=2,5,8,12,16$, and 20 $\mathrm{keV}$. For $k T_{\mathrm{e}}=20 \mathrm{keV}$ the dashed line indicates the calculation including only the recoil effect after scattering.

background intensity $F_{\nu}(\nu)=S_{\nu}(\nu) / h \nu$ in the form $F_{\nu}=A \nu^{-\alpha} \exp \left(-\nu / \nu_{0}\right)$ (the part of the spectrum from Eq. (1) corresponding to low energies $h \nu \lesssim 60 \mathrm{keV}$ ) into the right-hand side of this equation, for the relative changes in the background we find

$$
\begin{gathered}
\frac{\Delta F_{\nu}}{F_{\nu}}=\frac{h \nu}{m_{\mathrm{e}} c^{2}} \frac{\tau_{\mathrm{c}} \xi\left(T_{\mathrm{e}}\right)}{1+\beta \nu+\gamma \nu^{2}}\left[1-\frac{k T_{\mathrm{e}}}{h \nu_{0}}+\right. \\
\left.+\left(1-\alpha-\frac{\nu}{\nu_{0}}-\frac{\nu}{\nu_{1}}\right)\left(1-\frac{k T_{\mathrm{e}}}{h \nu_{0}}-\frac{k T_{\mathrm{e}}}{h \nu}(2+\alpha)\right)\right] .
\end{gathered}
$$

Here, we introduce the notation $\nu_{1}$ for the function

$$
\nu_{1}(\nu)=\frac{1+\beta \nu+\gamma \nu^{2}}{\beta+2 \gamma \nu} .
$$

The distortion spectrum for the harder, $h \nu \gtrsim 60 \mathrm{keV}$, (power-law) part of the spectrum can be found from Eq. (9) by passing to the limit $\nu_{0} \rightarrow \infty$ :

$$
\begin{gathered}
\frac{\Delta F_{\nu}}{F_{\nu}}=\frac{h \nu}{m_{\mathrm{e}} c^{2}} \frac{\tau_{\mathrm{c}} \xi\left(T_{\mathrm{e}}\right)}{1+\beta \nu+\gamma \nu^{2}} \times \\
\times\left[1+\left(1-\alpha-\frac{\nu}{\nu_{1}}\right)\left(1-\frac{k T_{\mathrm{e}}}{h \nu}(2+\alpha)\right)\right] .
\end{gathered}
$$

In the limit $\beta \nu \ll 1$ and $\nu \ll \nu_{0}$ Eq. (10) gives

$$
\frac{\Delta F_{\nu}}{F_{\nu}}=(2+\alpha) \tau_{\mathrm{c}} \frac{k T_{\mathrm{e}} \xi\left(T_{\mathrm{e}}\right)}{m_{\mathrm{e}} c^{2}}\left[\alpha-1+\frac{2-\alpha}{2+\alpha} \frac{h \nu}{k T_{\mathrm{e}}}\right] .
$$

Accordingly, to a first approximation, for $h \nu \lesssim k T_{\mathrm{e}}$, the amplitude of the effect is proportional to the Compton parameter $y_{\mathrm{C}}=\tau_{\mathrm{c}} \xi\left(T_{\mathrm{e}}\right) k T_{\mathrm{e}} / m_{\mathrm{e}} c^{2}$. In the opposite limit $h \nu \gg k T_{\mathrm{e}}$ the formula for the distortions takes the form

$$
\frac{\Delta F_{\nu}}{F_{\nu}}=-\tau_{\mathrm{c}} \xi\left(T_{\mathrm{e}}\right) \frac{h \nu}{m_{\mathrm{e}} c^{2}} \frac{\alpha-2+\nu / \nu_{1}}{1+\beta \nu+\gamma \nu^{2}},
$$

i.e., the amplitude of the effect is proportional to $\tau_{\mathrm{c}}$.

The thick solid (blue) lines in Fig.7 indicate the results of applying Eqs. (9) and (10) to a cluster with a radial optical depth $\tau_{\mathrm{c}}=6 \times 10^{-3}$ (which corresponds to the optical depth along the line of sight passing through the center $\tau_{\mathrm{T}}=1.2 \times 10^{-2}$ ) and electron temperatures $k T_{\mathrm{e}}=2,5,8,12$, and $20 \mathrm{keV}$. At $h \nu>60 \mathrm{keV}$ we used a superposition of the solutions (10) for the spectra with different photon indices in accordance with the model of the background spectrum (Eq. (1)). Since the background spectrum was fitted by different functions at low and high energies, its derivative can have a discontinuity at $60 \mathrm{keV}$. In the distortion spectra calculated from the approximate formulas (90) and (10), a jump or break is observed near this energy for many temperatures. Therefore, the analytical solution in the narrow region $\pm 10 \mathrm{keV}$ near $60 \mathrm{keV}$ is not shown in the figure for clarity. For clarity, we also disregard the photoabsorption of background photons by strongly ionized iron ions. This process can be easily included in the analytical solution (see Grebenev and Sunyaev 1987).

The thin (green) lines in Fig.7 indicate the results of our Monte Carlo computations of the distortions in the background spectrum. The computations were performed for the same cluster parameters as those for the analytical solution and allow them to be compared. However, they differ not only by the method of solution, but also by the boundary conditions: for the Kompaneets equation the isotropic background source is located at the gas cloud center, while for the Monte Carlo method the background radiation penetrates the cloud from outside. Note that an indistinct feature, which, obviously, is associated with the piecewise continuous fit of the background spectrum (Eq. (1)), is also observed near $60 \mathrm{keV}$ in many of the spectra computed by the Monte Carlo method. 

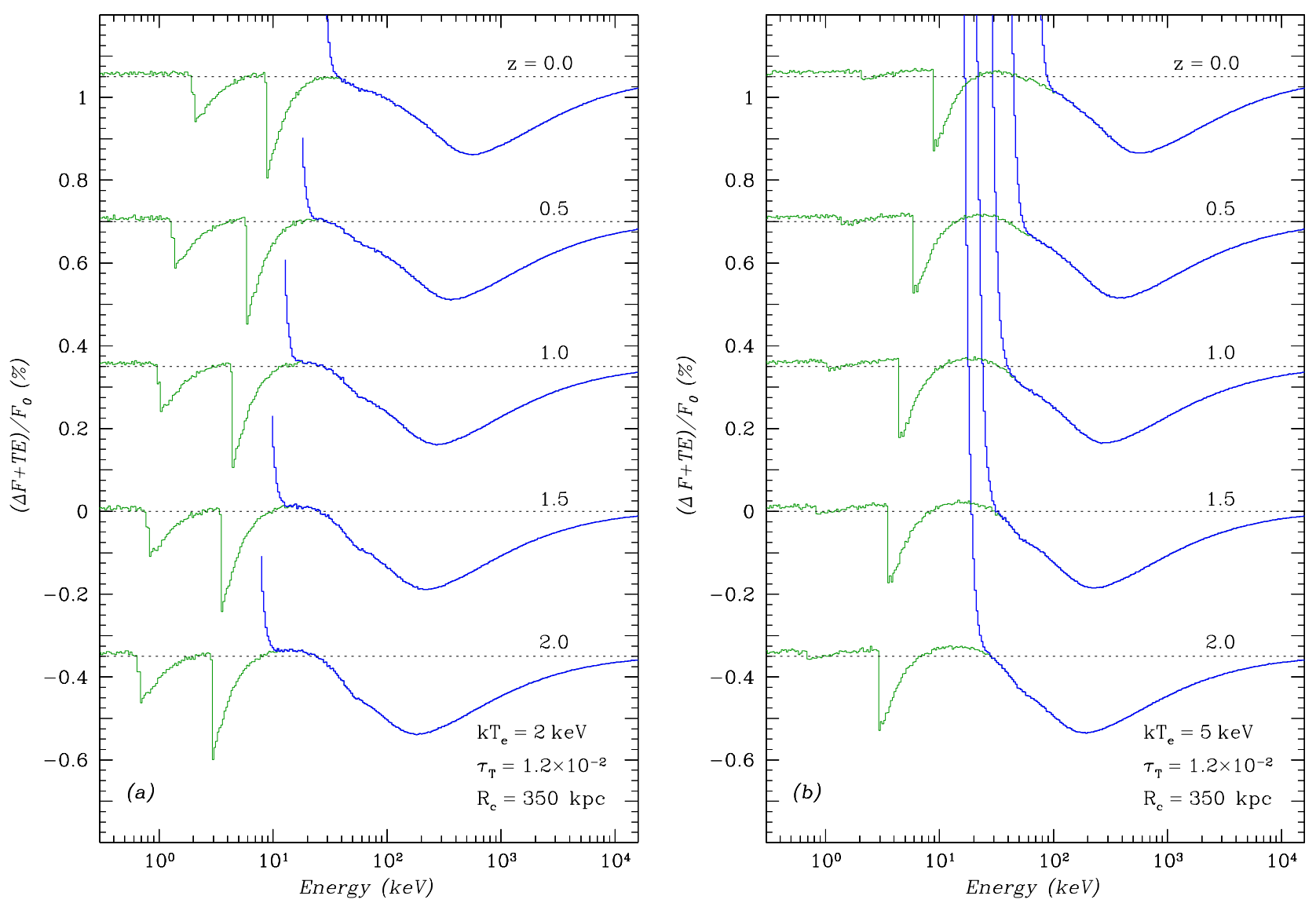

Fig. 8. Expected cosmic background (its intensity) distortions after scattering and absorption in the hot cluster gas versus its redshift $z$ (thin green lines). Clusters with a uniform density, optical depth $\tau_{\mathrm{T}}=1.2 \times 10^{-2}$, and core radius $R_{\mathrm{c}}=350 \mathrm{kpc}$. The gas temperature is $k T_{\mathrm{e}}=$ (a) 2 and (b) $5 \mathrm{keV}, Z=0.5 Z_{\odot}$. The thick (blue) lines indicate the background distortions including the thermal plasma radiation (shown incompletely on panel (a)).

On the whole, it can be said that the calculation based on Eqs. (9) and (10) correctly reproduces the spectral shape of the numerically computed distortions, although it smoothes the deepest part of the absorption feature at energies $h \nu \sim 500-600 \mathrm{keV}$ arising due to the recoil effect. It is possible that a better coincidence could be achieved here by numerically integrating the relativistic kernel of the kinetic equation for Compton scattering (Sazonov and Sunyaev 2000), but in this case an expression for the spectrum could not be obtained explicitly. The presented formulas give quite reasonable estimates of the distortions in the background spectrum. The analytical solution also allows the nature of the various components in the distortion spectrum to be easily clarified. For example, the dashed line in Fig.7 indicates our calculation of the distortions in the limit $T_{\mathrm{e}} \rightarrow 0$, i.e., arising in the background spectrum due to the recoil effect. Clearly, almost the entire excess of radiation at $h \nu \lesssim 150 \mathrm{keV}$ and the shape of the left edge of the absorption feature at $h \nu \gtrsim 150 \mathrm{keV}$ are associated with the Maxwellian velocities of electrons and are formed due to the Doppler effect.

\section{DEPENDENCE ON $Z$}

One of the remarkable properties of the effect of microwave background radiation scattering by the hot gas of galaxy clusters is its independence of the redshift $z$. Indeed, no matter how far the cluster is, the distortions are observed in its present-day well measured spectrum characterized by a temperature $T_{\mathrm{r}}=2.7 \mathrm{~K}$. Although the temperature $T_{\mathrm{r}}$ was a factor of $(1+z)$ higher at the time of its interaction with the cluster, this does not manifest itself in any way, because the equation describing the Doppler spectral distortions depends only on $x=h \nu / k T_{\mathrm{r}}$ (Zel'dovich and Sunyaev 1982), i.e., it is invariant in $z$.

The situation with the X-ray background scattering is different. Here all distortions are also observed in the present-day spectrum, although they are formed in the background spectrum at the cluster redshift $z$. However, apart from the Doppler ones, among them there are the distortions that arise due to photoabsorption and the recoil effect at quite specific energies - the absorption thresholds $h \nu \sim 2$ and $9 \mathrm{keV}$ and at $h \nu \sim 500 \mathrm{keV}$. In the spectrum observed at $z>0$ these features turn out to be 
shifted to low energies. Figure 8 presents the spectra of the background distortions that should be recorded from clusters with the same parameters $\left(\tau_{\mathrm{T}}=1.2 \times 10^{-2}\right.$, $R_{\mathrm{c}}=350 \mathrm{kpc}$, and $k T_{\mathrm{e}}=2 \mathrm{keV}$ in Fig.8 $8 \mathrm{a}$ or $5 \mathrm{keV}$ in Fig.8b), but located at different redshifts. The spectra were obtained by recalculating the present-day background spectrum (Eq. (1)) to the corresponding $z$ using standard formulas (see, e.g., Zel'dovich and Novikov 1975), computing the distortions there, and then recalculating the spectrum back to $z=23$. Both (photoabsorption and recoil effect) lines are seen to be actually greatly shifted leftward; at $z=2$ the absorption line is at $\sim 3 \mathrm{keV}$, while the minimum of the feature related to the recoil effect is at $\sim 200 \mathrm{keV}$. However, the case of $z=2$ is, in a sense, extreme - the clusters are subject to strong evolution and at $z \sim 2$ they (especially massive clusters) were much fewer than those observed now. Note that the Doppler spectral distortions (the excess of radiation), which clearly manifest themselves at low energies in Fig.8 $\mathrm{b}$, are almost independent of $z$, like the microwave background distortions.

As $z$ increases, the spectrum of the thermal plasma radiation in the cluster is also shifted to low energies and its intensity decreases. This is indicated by the thick (blue) lines in Fig.8. This shift (and attenuation) allows the effects of Compton scattering and photoabsorption of the background in distant clusters to be investigated at lower energies. The fundamental difference in the shape of the $z$ dependence of the amplitude of Compton X-ray background distortions compared to the flux of bremsstrahlung and recombination radiation is illustrated by Fig.9. The solid (dark green) lines in this figure indicate the variation in the spectral flux of thermal radiation wth $z$ for a cluster with a uniform density distribution, $\tau_{\mathrm{T}}=1.2 \times 10^{-2}, R_{\mathrm{c}}=350 \mathrm{kpc}$, and $k T_{\mathrm{e}}=2 \mathrm{keV}$ expected during its observation by a telescope with an aperture radius of $5^{\prime}$ (FWHM). The curves from top to bottom correspond to the fluxes at energies $h \nu=2,5,12$ and $20 \mathrm{keV}$. The dashed line indicates the $z$ dependence of the integrated flux. The initial flux rise up to $z \simeq 0.06$ is related to the increase in the volume emission measure of the cluster gas visible within the aperture:

$$
E M=\frac{4 \pi}{3} R_{\mathrm{c}}^{3}\left[1-\left(1-\frac{\rho_{\mathrm{z}}^{2}}{R_{\mathrm{c}}^{2}}\right)^{3 / 2}\right],
$$

where $\rho_{\mathrm{z}}$ is the impact parameter in the cluster frame corresponding to the specified aperture width. At high $z \mathrm{z}$, when the cluster is already completely within the field of view, the integrated flux of its thermal radiation drops with $z$ as a power law; the spectral fluxes at high energies drop more rapidly due to the cutoff of the bremsstrahlung and recombination radiation spectrum at the corresponding energies. The dotted line indicates

\footnotetext{
${ }^{3}$ In our calculations we adopted the standard $\Lambda \mathrm{CDM}$ cosmological model with $\Omega_{\mathrm{M}}=0.3, \Omega_{\Lambda}=0.7$, and $H=70 \mathrm{~km} \mathrm{~s}^{-1} \mathrm{Mpc}^{-1}$.
}

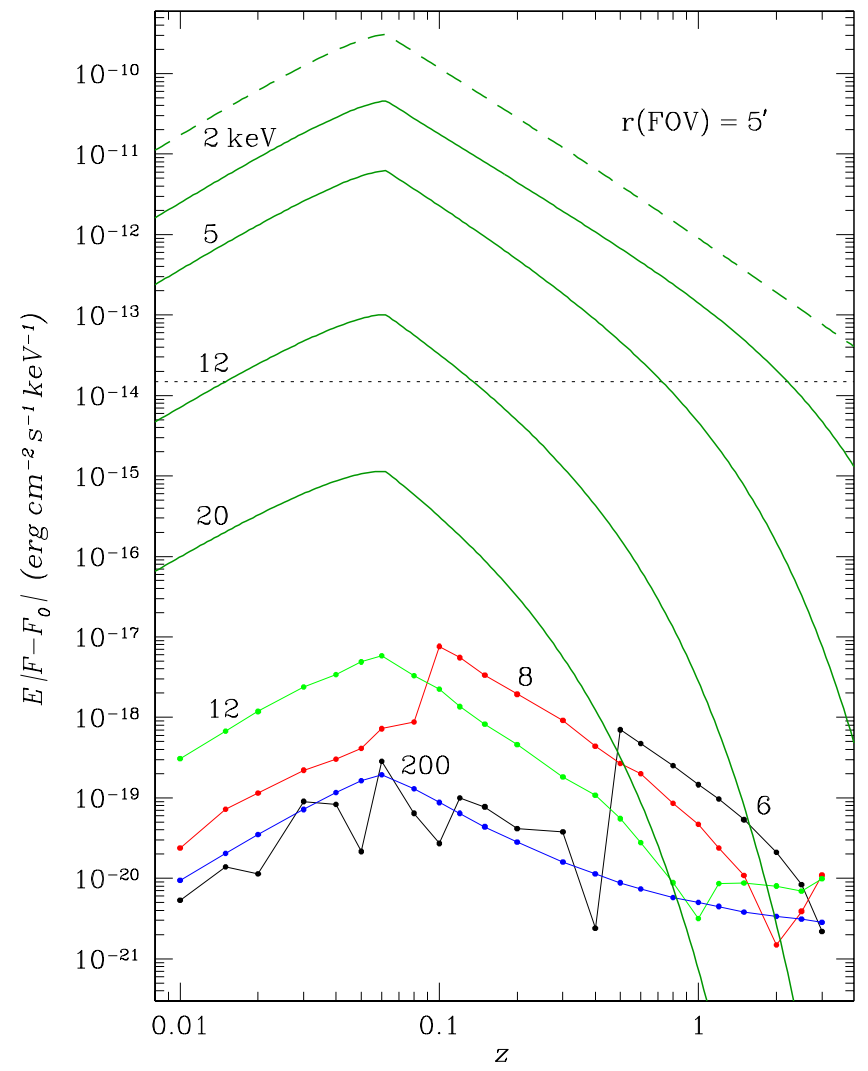

Fig. 9. Variations in the spectral flux (recorded within the telescope aperture with a $5^{\prime}$ radius) of thermal X-ray radiation from the hot gas in a galaxy cluster (dark green lines) and the absolute amplitude of the background distortions due to Compton scattering and photoabsorption in this gas (lines with dots) with $z$. The energy $h \nu$ for which the flux is given is specified near each curve. The dashed line indicates the change in the integrated flux of thermal radiation. The dotted line indicates the X-ray background flux at $5 \mathrm{keV}$. The cluster gas has an optical depth for Thomson scattering $\tau_{\mathrm{T}}=1.2 \times 10^{-2}$, core radius $R_{\mathrm{c}}=350 \mathrm{kpc}$, and temperature $k T_{\mathrm{e}}=2 \mathrm{keV}$.

the X-ray background flux at $5 \mathrm{keV}$ falling into this aperture.

Because of the shift of the photoabsorption lines and the absorption feature related to the recoil effect toward low energies, the $z$ dependence of the amplitude of the Compton distortions and photoabsorption takes a fairly complex shape (Fig.9, especially 6 and $8 \mathrm{keV}$ ). The abrupt jumps on these curves are associated with the passage of the threshold of the absorption line at $\sim 9 \mathrm{keV}$ as $z$ changes, given its fine structure. Such jumps should also be observed at low energies - leftward of the absorption threshold at $\sim 2 \mathrm{keV}$. We emphasize that this figure shows the amplitude of the absolute background distortions (i.e., the difference in the spectral flux of radiation within the aperture compared to the initial spectrum taken in absolute value). The 
Table 4. Parameters of the individual clusters selected to estimate the effect of scattering and absorption of the X-ray background in their hot intergalactic gas*

\begin{tabular}{|c|c|c|c|c|c|c|c|c|c|c|c|c|c|}
\hline \multicolumn{2}{|c|}{ Cluster name $^{\mathrm{a}}$} & \multirow[t]{2}{*}{$z$} & \multirow{2}{*}{$\begin{array}{c}R_{500},{ }^{\mathrm{b}} \\
\mathrm{kpc}\end{array}$} & \multirow{2}{*}{$\begin{array}{l}R_{\mathrm{c}} \\
\mathrm{kpc}\end{array}$} & \multirow{2}{*}{$\begin{array}{c}\theta_{\mathrm{c}} \\
,\end{array}$} & \multirow{2}{*}{$\begin{array}{l}k T_{\mathrm{e}}, \\
\mathrm{keV}\end{array}$} & \multirow[t]{2}{*}{$N_{\mathrm{c}}^{\mathrm{C}}$} & $M_{\mathrm{g}}$ & $M_{500} \mathrm{~b}$ & \multirow[t]{2}{*}{$Y_{\mathrm{SZ}}{ }^{\mathrm{d}}$} & \multirow{2}{*}{$\begin{array}{l}\mathrm{Z}^{\mathrm{e}}, \\
Z_{\odot}\end{array}$} & \multirow{2}{*}{$\begin{array}{c}\tau_{\mathrm{T}}{ }^{\mathrm{f}} \\
10^{-3} \\
\end{array}$} & \multirow[t]{2}{*}{ Reference $^{\mathrm{g}}$} \\
\hline main & alternative & & & & & & & \multicolumn{2}{|c|}{$10^{14} M_{\odot}$} & & & & \\
\hline 1 & 2 & 3 & 4 & 5 & 6 & 7 & 8 & 9 & 10 & 11 & 12 & 13 & 14 \\
\hline AT J0102-4915 & El Gordo & 0.870 & $\underline{950}$ & 270 & 0.75 & 14.5 & 8.9 & 2.2 & 11.9 & 32.0 & 0.2 & 15.2 & $1,2,3$ \\
\hline A 426 & Perseus & 0.018 & 1400 & 280 & 12.8 & 6.0 & $\overline{4.6}$ & 2.0 & $\underline{8.0}$ & 11.9 & 0.5 & $\underline{8.3}$ & $4,5,6$ \\
\hline ST J0615-5746 & P G266.6-27.3 & 0.972 & $\overline{1100}$ & $\underline{230}$ & 0.63 & 14.2 & $\underline{7.2}$ & 1.12 & $\overline{8.7}$ & $\overline{15.9}$ & 0.7 & $\overline{10.5}$ & $2,3,7,8$ \\
\hline $1 \mathrm{E} 0657-558$ & Bullet & 0.296 & 1660 & 170 & 0.73 & 12.4 & 12.3 & 2.01 & 12.7 & 24.9 & 0.3 & 13.3 & $2,3,7,9$ \\
\hline A 1367 & Leo & 0.022 & $\underline{760}$ & 210 & 8.1 & 3.7 & $\overline{1.1}$ & $\underline{0.11}$ & 1.3 & $\underline{0.4}$ & 0.5 & 1.2 & 5,6 \\
\hline A 1656 & Coma & 0.023 & $\overline{1310}$ & 290 & 10.5 & 6.9 & 2.9 & $\overline{1.0}$ & 3.5 & 6.7 & $\underline{0.5}$ & 5.6 & $6,10,11$ \\
\hline Virgo & & 0.004 & 770 & 310 & 62.5 & 2.4 & 2.7 & $\overline{1.5}$ & 8.0 & $\overline{3.6}$ & $\overline{0.3}$ & 5.3 & $12,13,14$ \\
\hline A 1991 & & 0.059 & 730 & 60 & 0.90 & 2.3 & 6.4 & 0.1 & 1.23 & $\overline{0.2}$ & $\underline{0.5}$ & 3.5 & $5,6,15$ \\
\hline ST J2106-5844 & & 1.132 & 960 & 200 & 0.54 & 9.4 & 11.5 & 1.17 & 7.1 & $\widetilde{11.1}$ & $\overline{0.3}$ & 14.6 & $2,3,7$ \\
\hline ST J2248-4431 & AS 1063 & 0.348 & 1630 & 370 & 1.4 & 11.5 & $\underline{2.9}$ & 1.95 & 13.1 & 22.3 & 0.3 & 7.0 & $2,3,7$ \\
\hline ST J2344-4243 & Phoenix & 0.596 & 1330 & 290 & 0.88 & 14.9 & 4.8 & 1.48 & 9.6 & 22.1 & 0.5 & 8.8 & $2,3,7,16$ \\
\hline
\end{tabular}

a A - Abell, ST - SPT-CL, AT - ACT-CL, P - PLCK.

$\mathrm{b}$ The radius bounding the mass $M_{500}$ of a cluster with a mean density equal to $500 \rho_{\mathrm{cr}}(z)$ of the Universe.

c The gas density at the cluster center, $10^{-3} \mathrm{~cm}^{-3}$.

d The microwave background scattering "efficiency" (Kravtsov et al. 2006), $10^{14} M_{\odot} \mathrm{keV}$.

e The abundance of the iron-group elements compared to the normal cosmic abundance.

$\mathrm{f}$ The Thomson optical depth along the line of sight passing through the cluster center.

g 1 - Menanteau et al. (2012); 2 - Bulbul et al. (2019); 3 - Bleem et al. (2015); 4 - White et al. (1997);

5 - Jones and Forman (1984); 6 - David et al. (1993); 7 - Williamson et al. (2011); 8 - Aghanim et al. (Planck Collaboration) (2011); 9 - Markevitch et al. (2002); 10 - Ade et al. (Planck Collaboration) (2013); 11 - Herbig et al. (1995); 12 - Forman and Jones (1982); 13 - Ade et al. (Planck Collaboration) (2016b); 14 - Gavazzi et al. (2009); 15 - Vikhlinin et al. (2006); 16 - McDonald et al. (2015).

* The underlined estimates were obtained using the formulas and dependences from Navarro et al. (1997) and Kravtsov et al. (2006).

previous figures presented the relative distortion amplitude (in \%). Here (just as above when considering the thermal cluster radiation) we took into account the effect of observation of only the part of the cluster at low $z$ (due to the excess of its size above the aperture size) and the effect of observation of a noticeable fraction of the undistorted background at high $z$ (because the cluster begins to occupy only part of the aperture). In clusters with a smooth density distribution (described by the $\beta$ or NFW models - see the Appendix) the curves in Fig.9 modified by these effects will be smoother.

As follows from the figure, the amplitude of the background distortions due to scattering and absorption changes with $z$ much more weakly than the flux of thermal radiation. In this case, the probability of detecting the distortions in the background spectrum from the interaction with the gas of distant clusters may turn out to be even higher than that from the interaction with the gas of nearby clusters. In any case, the detection of such distortions remains a very challenging problem.

\section{INDIVIDUAL CLUSTERS}

Table 4 gives basic characteristics (the temperature and central density of the intergalactic gas, other parameters of the $\beta$ model density distribution) for several known rich clusters that exhibit strong microwave background radiation distortions. In particular, this can be seen from the high values of the parameter $Y_{\mathrm{SZ}}$ characterizing the amplitude of the background distortions (Kravtsov et

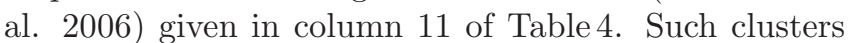
as Phoenex, SPT-CL J0615-5746, SPT-CL J2106-5844, and El Gordo were even discovered owing to this effect — by SPT (Williamson et al. 2011), ACT (Menanteau et al. 2012), or the Planck satellite (Aghanim et al. (Planck Collaboration), 2011). These are mostly very massive hot clusters with $k T_{\mathrm{e}} \gtrsim 10 \mathrm{keV}$, but the cold nearby Virgo, A 1367, and A 1991 clusters were also included in the list. The detectability of distortions in the $\mathrm{X}$-ray background from a cluster, and this list was compiled precisely for its estimation, is determined by many factors, and a reliable detection of the effect in the microwave background by no means implies that it can be detected in X-rays.

For a number of clusters we failed to find the measured values of some density distribution parameters. These parameters were then estimated from their dependence on $M_{500}\left(Y_{\mathrm{SZ}}\right)$ determined by Vikhlinin et al. (2006) and Kravtsov et al. (2006). In Table 4 their values are underlined for clarity. For all clusters we calculated the 


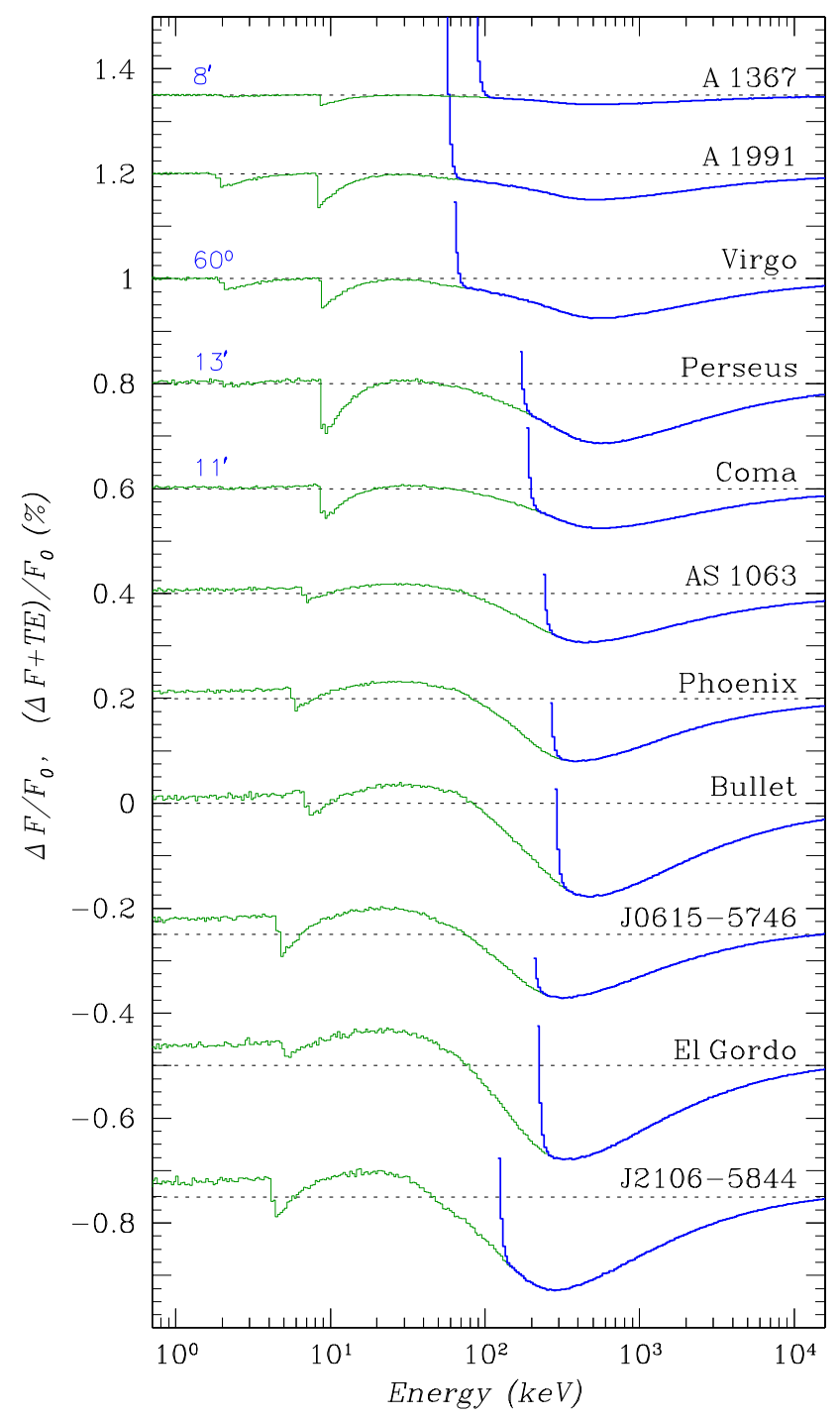

Fig. 10. Expected distortions in the spectrum of the X-ray background intensity due to Compton scattering and photoabsorption in the hot gas of several known galaxy clusters (thin green lines). The thick (blue) lines also take into account the thermal gas radiation.

optical depth for Thomson scattering along the line of sight passing through their center (column 13 in the table). Note that for such nearby and extended clusters as Virgo, Coma, and Perseus it turns out to be only a factor of 2-3 smaller than the optical depth of distant supermassive clusters like El Gordo, Bullet, SPT-CL J21065844, and SPT-CL J0615-574. For convenience, column 6 gives the angular sizes of the clusters corresponding to the values of $R_{\mathrm{c}}$ specified in Table 4 . For all clusters in the table we computed the relative distortions produced by them (the gas kept by their gravity) in the $\mathrm{X}$ ray background radiation spectrum by the Monte Carlo method under the assumption of a $\beta$ density distribution of the intergalactic gas. The computations were carried out for the same exponent of the distribution $\beta=2 / 3$, because the scatter of $\beta$ values for separate clusters did not exceed the errors of their determination. The abundance of the iron-group elements $Z$ in the gas relative to the cosmic abundance (column 12 in the table) was taken from the literature. The cluster redshifts were taken into account.

The results of our computations of the relative background radiation distortions (in percent to the initial spectrum) are presented in Fig.10. They correspond to the observations toward the cluster center by a telescope with a narrow aperture corresponding to the cluster core radius ( $\sim 1^{\prime}$ for most clusters, see Table 4$)$. As one recedes from the cluster center or when observing by a telescope with a wide aperture, the distortion amplitude should drop. The thin (green) and thick (blue) lines indicate the background distortions proper and the distortions including the thermal gas radiation, respectively. We see that the rich hot clusters in the lower part of the figure lead to large deviations of the background over the entire spectrum - positive at $h \nu \lesssim 100 \mathrm{keV}$ (the excess radiation due to the Doppler effect) and negative at energies above $h \nu \gtrsim 100 \mathrm{keV}$ (the dip in the spectrum due to the recoil effect). The feature at $\sim 9 \mathrm{keV}$ associated with photoabsorption by iron is noticeably weaker in amplitude than the MeV dip — the iron at temperatures typical for such clusters is almost fully ionized. We also see that the intrinsic thermal gas radiation presents a huge problem for the detection of the effect being discussed at energies $\lesssim 200 \mathrm{keV}$.

For the cold clusters in the upper part of the figure, such as Virgo, A 1991, Coma, and Perseus, the amplitude of the positive background distortions at low energies is negligible, while the amplitude of the MeV dip remains fairly large; it differs from the amplitude of the dip for the SPT-CL J2106-5844, SPT-CL J0615-5746, and El Gordo clusters, which were believed to be most massive in the Universe, only by a factor of 2 or 3 . The features due to photoabsorption in the spectrum of the background distortions by these clusters are expectedly larger than those for the hot clusters due to the low gas temperature - the feature at $9 \mathrm{keV}$ is even comparable in amplitude to the dip at high energies. The thermal radiation from the clusters, also expectedly, begins to hinder the detection of the effect at appreciably lower $(h \nu \lesssim 50$ $\mathrm{keV}$ ) energies than that for the rich clusters. The figure suggests that for the Virgo and A 1991 clusters the drop in the background due to recoil effect can be searched for already at energies $60-100 \mathrm{keV}$ without any noise related to the thermal gas radiation. It is important that the Virgo cluster, along with Perseus, Coma, and A 1367 have noticeable angular sizes (see the left part of Fig.10, where the angular core radius of these clusters is presented in arcmin/deg). The observations of such extended clusters even at a slightly smaller amplitude of the background distortions than that for rich, but distant clusters may turn out to be much more significant and fruitful. 


\section{X-RAY BACKGROUND FLUCTUATIONS}

The detection of hard X-ray background deviations at a level of fractions of percent is not something absolutely unattainable per se. Such measurements aimed at searching for background fluctuations have already been carried out by both HEAO-1 (Boldt 1987; Treyer et al. 1998) and RXTE (Gruber et al. 1999b; MacDonald et al. 2001) observatories.

In particular, the HEXTE/RXTE instrument performed almost simultaneous observations of two sky regions spaced $3^{\circ}$ apart. The differences in the fluxes measured in these regions within the field of view of the instrument with an area of $1.1 \mathrm{sq}$. deg allowed background fluctuations much weaker than was possible in individual observations to be searched for. Such fluctuations were actually detected at a flux level of $(0.092 \pm 0.014)$, $(0.11 \pm 0.02)$, and $(0.23 \pm 0.08) \%$ of the background spectrum in the energy ranges $15-20,20-25$, and $34-41 \mathrm{keV}$, respectively. The relative amplitude of the fluctuations is seen to have increased with energy. Similar results, in both background fluctuation level and their energy dependence, were previously obtained by the A-2 instrument of the HEAO-1 observatory.

The authors explained their results by a nonuniform distribution of matter in the Universe and, accordingly, by a nonuniform distribution of AGNs, which are to a large extent responsible for the hard X-ray background. The energy dependence of the fluctuation level can then be explained by the drop in the effective number of sources responsible for the background and fluctuations when passing to higher energies. However, it may well be that not only AGNs, but also the distortions forming in the spectrum of the background radiation as it passes through the hot intergalactic gas in unresolved distant clusters of galaxies were one of the causes of the detected background fluctuations. At least the relative amplitudes of the detected fluctuations agree well with the results of our computations presented in this paper.

Therefore, the conclusion reached by Boughn and Crittenden (2004, 2005) about a significant correlation of the fluctuations in the distribution of the X-ray background measured by the HEAO-1 observatory and the microwave background measured by the WMAP satellite is also of interest. It seem quite probable that in both energy ranges we are dealing with the fluctuations associated with the same phenomenon - the cosmic background scattering in the hot gas of clusters of galaxies. Estimates and detailed computations of the role of distant clusters in the formation of the microwave background fluctuations have been performed at some time by Longair and Sunyaev (1969) and Markevitch et al. (1992).

The fact of achieving such a high sensitivity when observing the X-ray background fluctuations in wide sky fields confirms that the study is important and promising for detecting the effect being discussed in nearby extended galaxy clusters, such as Virgo, Coma, A 426, and A 1367.

\section{SCATTERING OF RADIATION FROM ACTIVE GALAXIES OF A CLUSTER}

Some of the galaxy clusters may have active nuclei (AGNs), whose hard radiation should be subject to scattering and absorption in its hot gas, like the background radiation. The scattered diffuse X-ray radiation from such an AGN can be perceived as an additional background distortion.

Can this radiation hinder the detection of the effect being discussed in the paper? Let us place an AGN with an X-ray luminosity $L_{\mathrm{X}}=1 \times 10^{43} \mathrm{erg} \mathrm{s}^{-1}$ and a power-law spectrum with an exponential cutoff at high energies typical for AGNs at the center of our standard model cluster with a uniform density, optical depth $\tau_{\mathrm{T}}=1.2 \times 10^{-2}$, core radius $R_{\mathrm{c}}=350 \mathrm{kpc}$, electron temperature $k T_{\mathrm{e}}=5 \mathrm{keV}$, and metallicity $Z=0.5 Z_{\odot}$. We will take the photon index of the spectrum to be $\alpha=1.9$ and the cutoff energy to be $E_{\mathrm{c}}=300 \mathrm{keV}$ (Sazonov et al. 2008; Ueda et al. 2014). The radiation spectrum of such an AGN is indicated in Fig. 11a by the dotted red line for comparison with the spectra of the hot gas in the galaxy cluster and the cosmic background radiation. Note that the X-ray luminosity of the thermal radiation from the cluster gas is $L_{\mathrm{c}}=6.9 \times 10^{44} \mathrm{erg} \mathrm{s}^{-1}$. The green line at the bottom in Fig.11b indicates the spectrum of the relative distortions arising in the AGN radiation when it passes through the hot cluster gas. It is very similar to the spectrum of the relative distortions arising in the background radiation (indicated in the same figure by the black line). Note only the shape of the MeV dip slightly skewed to low energies, which, obviously, reflects the difference in shape between the initial AGN radiation spectrum and the background spectrum (primarily the exponential cutoff at high energies in the AGN spectrum).

In reality, however, we are interested not in the distortions in the AGN radiation spectrum, but in its radiation that was scattered in the cluster and became diffuse, because the direct escape radiation will be perceived as the radiation of a compact source (AGN) when analyzing the data and, naturally, should be subtracted.

The blue line in Fig.11b indicates the spectrum of scattered AGN photons (relative to its initial spectrum), while the red dotted line indicates the same spectrum, but relative to the initial background radiation spectrum. Remarkably, the scattered photon spectrum is smooth and does not contain any negative features related to photoabsorption or the recoil effect after scattering. Its amplitude relative to the initial AGN radiation spectrum is more than $0.6 \%$, i.e., it is much greater than the amplitude of the final AGN radiation distortions. We noted these properties of the scattered radiation (only that of the background) previously when 


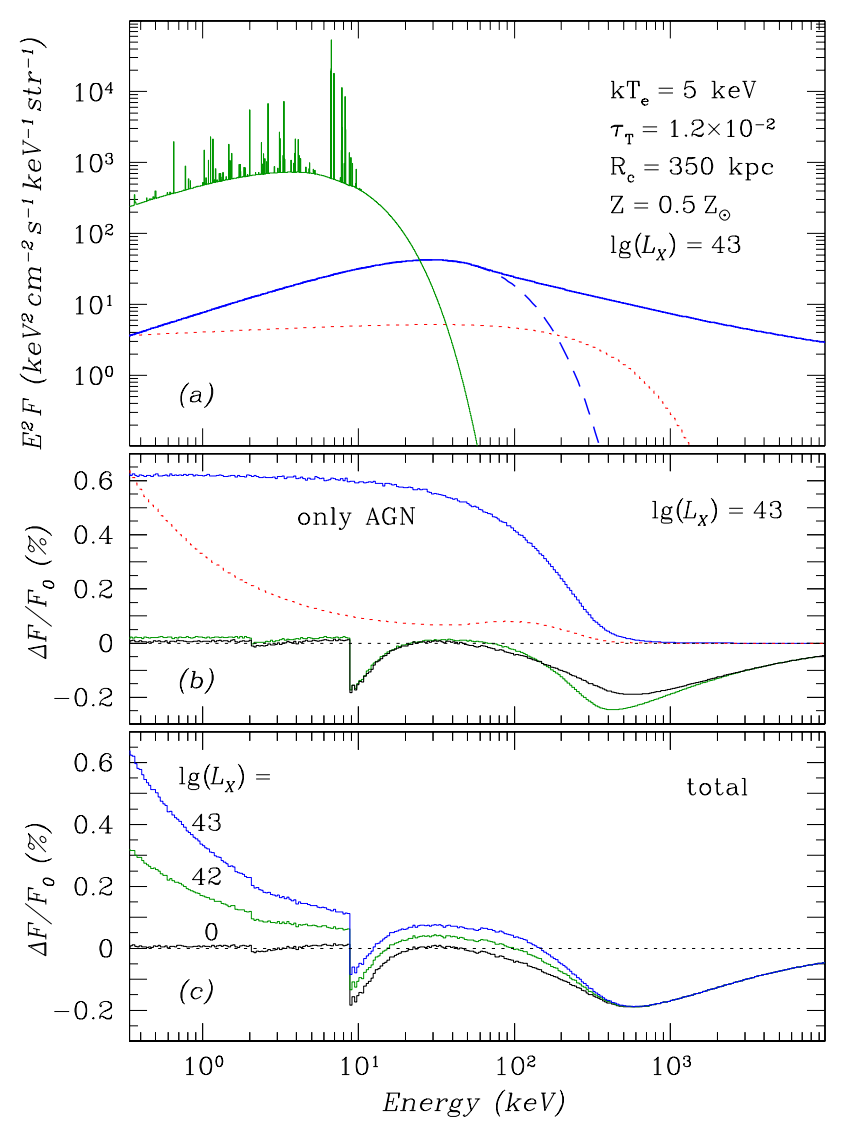

Fig. 11. Influence of the AGN radiation on the observations of background distortions in the hot cluster gas: (a) the AGN radiation spectrum (red dotted line) in comparison with the background and cluster gas spectra; (b) the distortions of the AGN (green) and background (black curves) spectra, the scattered AGN radiation relative to its initial spectrum (blue curve) and relative to the initial background spectrum (red dotted line); (c) the background distortions including the scattered AGN radiation. The optical depth of the gas toward the center is $\tau_{\mathrm{T}}=1.2 \times 10^{-2}$, the cluster radius is $R_{\mathrm{c}}=350 \mathrm{kpc}, k T_{\mathrm{e}}=5 \mathrm{keV}$, and $Z=0.5 Z_{\odot}$. The AGN $\mathrm{X}$-ray luminosity is $L_{\mathrm{X}}=1 \times 10^{43} \mathrm{erg} \mathrm{s}^{-1}$, the green line on the lower panel indicates the case of $L_{\mathrm{X}}=1 \times 10^{42} \mathrm{erg} \mathrm{s}^{-1}$.

discussing Fig.2 The amplitude of the scattered AGN radiation relative to the background spectrum turns out to be small everywhere, except the standard X-ray band $h \nu \lesssim 10 \mathrm{keV}$. The enhancement of the distortions in the soft X-ray band is related to the differences in the AGN and background spectra: the AGN intensity here begins to approach and even exceed the background intensity. However, many AGNs exhibit a low-energy cutoff in the radiation spectrum related to the absorption of their radiation in the gas-dust torus surrounding the supermassive black hole in the galactic nucleus. Clearly, when the absorption is taken into account, the rise of the relative background distortions due to the soft X-ray AGN radiation should be less distinct or even disappear altogether.
The important thing is that the spectrum of the scattered AGN radiation contains no absorption features and, therefore, it cannot reduce or smear such features in the spectrum of the background distortions arising when it passes through the hot cluster gas. In the case of very bright AGN flares occurring on a time scale of hundreds of thousands of years, narrow lines associated with resonance scattering of the AGN X-ray emission by Fe, S, and $\mathrm{Si}$ ions can appear in the background spectrum at these energies (Sazonov et al. 2002).

Indeed, as Fig.11k shows, adding the AGN radiation scattered in the cluster gas to the spectrum of the emerged background distortions does not smear the features related to background photoabsorption on the $K$ and $L$ shells of strongly ionized iron and nickel ions in the cluster gas. What is especially remarkable, the scattered AGN radiation does not reduce and does not smear the $\mathrm{MeV}$ dip in the background spectrum related to the recoil effect when its photons are scattered by electrons in the cluster gas.

At the same time, the AGN radiation can lead to a rise in background intensity at low energies $h \nu \lesssim 9 \mathrm{keV}$ and in the hard X-ray range $20-150 \mathrm{keV}$, making it difficult to correctly interpret the background distortions at these energies. What is especially bad, the scattered AGN radiation is subject to the X-ray echo effect (Fabian 1977; Vainshtein and Sunyaev 1980), which leads to the conservation of the scattered X-ray radiation from the AGN for many $\left(\Delta t \sim R_{\mathrm{c}} / c \simeq 1.1 \mathrm{Myr}\right.$, where $c$ is the speed of light) years after the decay of the X-ray activity of the AGN itself. Similar radiation of the former activity of the black hole at the center of our Galaxy was observed from molecular clouds (Markevitch et al. 1993; Sunyaev et al. 1993). On the other hand, at such a long time of the reaction to AGN activity $(\simeq 1 \mathrm{Myr})$, it will also take a long time for the scattered radiation to be completely formed and to reach a stable intensity level. Thus, the influence of the AGN radiation can be effectively below our estimates.

Obviously, our analysis is completely extended to the case where the AGN is located not inside the cluster, but at some distance behind it or even near it; it is only necessary to take into account the area of the solid angle at which the cluster is seen from the AGN location.

\section{BACKGROUND DISTORTIONS IN A WARM INTERGALACTIC MEDIUM}

At $z \lesssim 1$ the total mass of the visible matter contained in the stars, dust, and hot gas of galaxy clusters and the clouds of atomic and molecular hydrogen accounts for only $\sim 1 / 3$ of the entire baryonic mass of the Universe (Fukugita et al. 1998). At the same time, observations of the "forest" of $L y_{\alpha}$ hydrogen lines at $z \sim 3$ give a factor of $\sim 3$ greater value consistent with the mean baryon density in the Universe $\Omega_{\mathrm{B}}=0.044$ (Rauch 1998). It is believed that $\sim 2 / 3$ of the baryons that have eluded ob- 


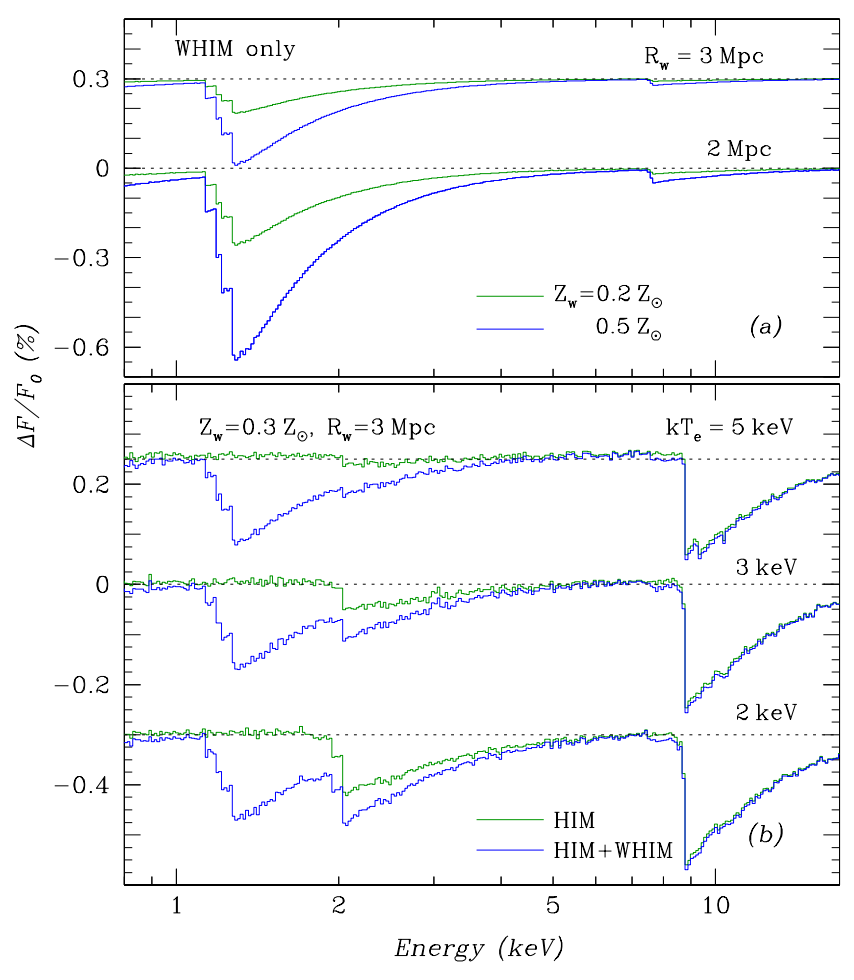

Fig. 12. Expected distortions in the X-ray background spectrum due to scattering and absorption in the envelope of the warm-hot intergalactic medium (WHIM) surrounding the galaxy cluster toward its center: (a) without the background distortions in the hot cluster gas and (b) with the distortions (the green lines indicate the background spectra before the distortion in WHIM). The hot gas (HIM) in the cluster has a Thomson optical depth toward the center $\tau_{\mathrm{T}}=1.2 \times 10^{-2}$, core radius $R_{\mathrm{c}}=350 \mathrm{kpc}$, electron temperature $k T_{\mathrm{e}}=2,3,5 \mathrm{keV}$, and metallicity $Z=0.5 Z_{\odot}$; the WHIM envelope has a factor of 4 larger mass, radius $R_{\mathrm{w}}=2$ or $3 \mathrm{Mpc}$, electron temperature $k T_{\mathrm{w}}=0.2 \mathrm{keV}$, and metallicity $Z_{\mathrm{w}}=0.2,0.3$, or $0.5 Z_{\odot}$.

servation are contained in the moderately hot plasma with a temperature $\sim 10^{6} \mathrm{~K}$ located in filaments and other similar structures on the far periphery of galaxy clusters (Cen and Ostriker 1999). This gas phase is called the Warm-Hot Intergalactic Medium (WHIM). The existence of WHIM was confirmed by the detection of a soft X-ray excess from a plasma with $k T \sim 0.2$ $\mathrm{keV}$ with a prominent blend of the O VII triplet at 0.57 $\mathrm{keV}$ and the O VIII $L y_{\alpha}$ line at $0.65 \mathrm{keV}$ in the spectra of several galaxy clusters (see, e.g., Finoguenov et al. 2003; Kaastra 2004a, 2004b).

Can the interaction of the X-ray background with WHIM give rise to additional distortions in its spectrum? Suppose, for simplicity, that each galaxy cluster is surrounded by a thick spherical WHIM layer (shell) with a quasi-uniform density distribution $\rho_{\mathrm{w}}=N_{\mathrm{w}} m_{\mathrm{p}}$. The outer radius of the layer is $R_{\mathrm{w}} \simeq 2-3 \mathrm{Mpc}$ (Kaas- tra 2004a). Assuming that the hot gas in the cluster (with a mass $M_{\mathrm{g}}$ within the radius $R_{\mathrm{c}}$ ) contains $\sim 50 \%$ of the visible matter, we find that the WHIM mass in the outer layer is $M_{\mathrm{w}} \simeq\left(2 / 3 \Omega_{\mathrm{B}}\right) /\left(0.5 \times 1 / 3 \Omega_{\mathrm{B}}\right) M_{\mathrm{g}} \simeq$ $4 M_{\mathrm{g}}$. The mean electron density in this layer is then $N_{\mathrm{w}}=4 N_{\mathrm{e}}\left(R_{\mathrm{c}} / R_{\mathrm{w}}\right)^{3} \simeq 5.3 \times 10^{-5} \times$

$$
\left(\frac{\tau_{\mathrm{T}}}{1.2 \times 10^{-2}}\right)\left(\frac{R_{\mathrm{c}}}{350 \mathrm{kpc}}\right)^{2}\left(\frac{R_{\mathrm{w}}}{3 \mathrm{Mpc}}\right)^{-3} \mathrm{~cm}^{-3} \text {. }
$$

This estimate agrees well with the WHIM density measurements from the O VII and O VIII lines in the X-ray spectra of galaxy clusters (Kaastra 2004a). The electron density in the hot cluster gas $N_{\mathrm{e}}$ expressed here via its Thomson optical depth along the line of sight passing through the cluster center, $\tau_{\mathrm{T}}=2 \sigma_{\mathrm{T}} N_{\mathrm{e}} R_{\mathrm{c}}=1.2 \times 10^{-2}$. The Thomson optical depth of the WHIM envelope along the same line of sight is $\tau_{\mathrm{w}} \simeq 2 \sigma_{\mathrm{T}} N_{\mathrm{w}}\left(R_{\mathrm{w}}-R_{\mathrm{c}}\right) \simeq$ $4 \tau_{\mathrm{T}}\left(R_{\mathrm{c}} / R_{\mathrm{w}}\right)^{2} \simeq 6.5 \times 10^{-4} \times$

$$
\left(\frac{\tau_{\mathrm{T}}}{1.2 \times 10^{-2}}\right)\left(\frac{R_{\mathrm{c}}}{350 \mathrm{kpc}}\right)^{2}\left(\frac{R_{\mathrm{w}}}{3 \mathrm{Mpc}}\right)^{-2} .
$$

Obviously, the distortions related to purely Compton scattering in WHIM will be the same as those after scattering in the hot cluster gas (Hot Integrgalactic Medium or HIM), but smaller in absolute value, because the optical depth of the envelope is small. In this case, the iron and nickel atoms in WHIM will be ionized much more weakly than those in the hot cluster gas (at $k T_{\mathrm{w}} \simeq 0.2$ $\mathrm{keV}$ - to Fe XIV-Fe XVII and Ni XIII-Ni XVII, respectively). Therefore, the photoabsorption of the background radiation in the envelope should be more efficient. Unfortunately, WHIM is most likely much poorer in metals than even HIM (see, e.g., Finoguenov et al. 2003); therefore, it is difficult to estimate this efficiency without numerical computations.

Figure 12 presents the results of such computations of the distortions in the X-ray background spectrum toward the cluster center arising when it passes through the WHIM envelope surrounding the cluster. The distortions only in WHIM are shown at the top (in Fig.12a); the changes in the spectrum that occurred in the hot cluster gas were disregarded here. It will be possible to see the shown distortions in cluster observations at large impact parameters $\rho \gg R_{\mathrm{c}}$. We considered the envelopes with various outer radii $R_{\mathrm{c}}$ (and, accordingly, various densities $N_{\mathrm{w}}$ and optical depths $\tau_{\mathrm{w}}$ ) and metallicities $Z_{\mathrm{w}}$. The WHIM temperature was taken to be $k T_{\mathrm{e}}=0.2 \mathrm{keV}$. The absorption threshold on the $K$ shell of iron and nickel is seen to be shifted leftward along the energy axis compared to the spectrum of the distortions in HIM (from $\simeq 8.8$ to $\simeq 7.6 \mathrm{keV}$ ); the absorption threshold on the $L$ shell is shifted even more strongly from $\simeq 2.03$ to $\simeq 1.27 \mathrm{keV}$. Whereas the hard line has a negligible depth, the depth of the absorption line at 1.27 $\mathrm{keV}$ is unexpectedly large even for the lowest metallicity considered $Z_{\mathrm{w}}=0.2 Z_{\odot}$. 


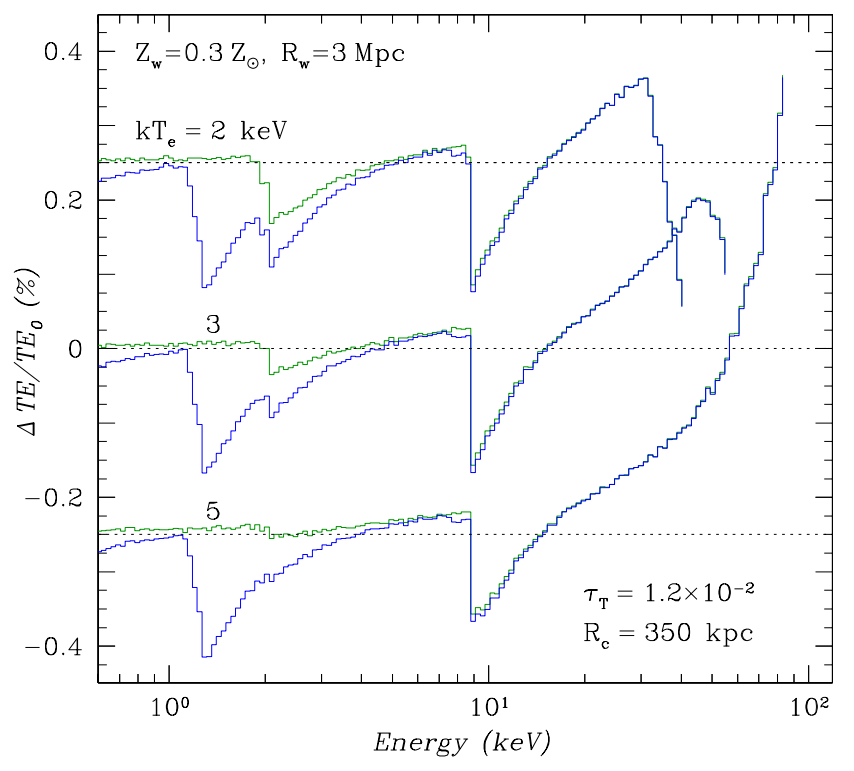

Fig. 13. Expected distortions in the thermal radiation spectrum of the hot cluster gas (HIM) in a galaxy cluster due to scattering and absorption in the envelope of the warmhot intergalactic medium (WHIM) surrounding the galaxy cluster toward its center (blue lines). The hot gas (HIM) in the cluster has a Thomson optical depth toward the center $\tau_{\mathrm{T}}=1.2 \times 10^{-2}$, radius $R_{\mathrm{c}}=350 \mathrm{kpc}$, electron temperature $k T_{\mathrm{e}}=2,3,5 \mathrm{keV}$, and metallicity $Z=0.5 Z_{\odot}$; the WHIM envelope has a factor of 4 larger mass, radius $R_{\mathrm{w}}=3$ Mpc, optical depth $\tau_{\mathrm{w}} \simeq 6.5 \times 10^{-4}$, electron temperature $k T_{\mathrm{w}}=0.2 \mathrm{keV}$, and metallicity $Z_{\mathrm{w}}=0.3 Z_{\odot}$. The green lines indicate the cluster HIM spectra before the interaction with WHIM.

Absorption lines are also formed at energies below $\sim 1 \mathrm{keV}$, but they are impossible to observe due to the intrinsic thermal radiation from WHIM. Most of the lines of resonant scattering of the X-ray background in WHIM predicted by Churazov et al. (2001) also fall into this region. Above $\sim 1 \mathrm{keV}$ there are only narrow recombination emission lines at energies $1.07,1.13,1.33-1.35$, and $1.87 \mathrm{keV}$ in the WHIM radiation spectrum. Note that iron and nickel ions with vacancies on the lower electronic shells are formed as a result of background photoabsorption in the WHIM plasma, which could not be formed in it due to collisional processes. Although these ions recombine mainly through the Auger effect, the formation of photons of fluorescent lines (similar to the $K_{\alpha}$ line with energy $6.4 \mathrm{keV}$ emitted with a $34 \%$ probability when neutral iron atoms are ionized) is also possible. There are no such emission lines in the intrinsic thermal recombination spectrum of WHIM. These lines are narrow and much harder to detect than the photoabsorption lines; therefore, they are disregarded in this paper.

The total spectra including the distortions in both
WHIM and HIM are presented at the bottom (in Fig.12b). The radius of the WHIM envelope was taken to be $R_{\mathrm{c}}=3 \mathrm{Mpc}$, the temperature is again $k T_{\mathrm{w}}=0.2$ $\mathrm{keV}$, and the metallicity is $Z_{\mathrm{w}}=0.3 Z_{\odot}$. The optical depth of the hot cluster gas is $\tau_{\mathrm{T}}=1.2 \times 10^{-2}$, the temperature is $k T_{\mathrm{e}}=2,3$, or $5 \mathrm{keV}$, and its metallicity is $Z=0.5 Z_{\odot}$. The spectra of the background distortions formed in HIM were previously presented in Fig.4. Substantial changes are seen to occur in the background spectrum when passing through the WHIM envelope: (1) a new intense broad absorption line appears at $\sim 1.3 \mathrm{keV}$, which does not depend on the cluster hot gas temperature; (2) the depth (equivalent width) of the absorption line at $\sim 2 \mathrm{keV}$ increases noticeably; and (3) the absorption threshold at $\sim 8.8 \mathrm{keV}$ is smeared.

Figure13 shows how the effect being discussed affects the spectrum of the intrinsic thermal (to be more precise, bremsstrahlung) plasma radiation. Our computations were done for the WHIM envelope and the hot cluster gas with the same parameters as those in Fig.12. The changes in the thermal spectra related to the interaction directly with the cluster gas (HIM) have already been shown in Fig.6. Here they are represented by the thin green lines. A broad absorption line with the threshold at $\sim 1.3 \mathrm{keV}$ and an amplitude of $\sim 0.16-0.22 \%$ (relative to the initial undistorted thermal spectrum) additionally appears in these spectra after the interaction with WHIM. Furthermore, the line at $\sim 2 \mathrm{keV}$ already present in the spectra of cold clusters is enhanced. The profile and amplitude of the absorption line at $\sim 9 \mathrm{keV}$ barely change. Thus, for favorable WHIM parameters two absorption lines at once, at $\sim 1.3$ and $9 \mathrm{keV}$, should be present in the thermal radiation spectra of the hot cluster gas.

The model used may be oversimplified and contains a number of poorly known parameters (primarily those related to the nonuniformity of the WHIM distribution). At the same time, it clearly shows that the interaction with WHIM can introduce noticeable distortions both into the cosmic X-ray background spectrum and the thermal radiation spectrum of the cluster, distortions comparable to those from their scattering and absorption in the hot cluster gas. This effect cannot not be neglected for nearby $(z \lesssim 1)$ clusters. The formation of the absorption line at $\sim 1.3 \mathrm{keV}$, whose amplitude does not depend on the hot gas parameters, but only on the WHIM parameters, may turn out to be very important in light of studying the properties of this mysterious matter itself in the distant environment of clusters of galaxies.

\section{MAIN RESULTS}

We presented the results of our numerical (Monte Carlo) and analytical (solving the Kompaneets equation) computations of the distortions arising in the spectrum of the X-ray and soft gamma-ray background radiation as 
it passes through the hot intergalactic gas in galaxy clusters. We investigated the dependence of the distortion amplitude and shape on the parameters of the cluster gas - its temperature, optical depth, and density distribution. The analogous distortions arising in the microwave background radiation are well known, are actively studied, and are widely used in observational cosmology. We showed the following:

1. Compton scattering by electrons of the intergalactic gas in clusters leads to peculiar distortions of the background radiation - a rise in its brightness at $h \nu \lesssim 60-100 \mathrm{keV}$ due to the Doppler effect and its drop at higher energies due to the recoil effect; the detection of background distortions allows the most important parameters of clusters and cosmological parameters to be measured;

2. the rise in background brightness in the hard Xray range is proportional to the Compton parameter $y_{\mathrm{C}}=\tau_{\mathrm{T}} k T_{\mathrm{e}} / m_{\mathrm{e}} c^{2}$, averaged over the visible (within the telescope aperture) part of the cluster (or the parameter $Y_{\mathrm{SZ}}$ in the case of a distant cluster), for the hottest clusters with $k T_{\mathrm{e}} \sim 15 \mathrm{keV}$ it reaches $\sim 0.1 \%$ at energies $\sim 100 \mathrm{keV}$;

3. the decrease in background brightness due to the recoil effect has a maximum at energies $\sim 500-600$ $\mathrm{keV}$ (in the cluster rest frame) and is proportional to the gas surface density or its Thomson optical depth $\tau_{\mathrm{T}}$ averaged over the visible (in the aperture) part of the cluster, it has an amplitude of $\sim 0.2-$ $0.3 \%$ for the optically thickest clusters and does not depend on the temperature;

4. photoabsorption by strongly ionized ions of the irongroup elements also leads to a decrease in the background with the formation of two absorption lines of characteristic shape with threshold energies $h \nu \sim 2$ and $9 \mathrm{keV}$ (in the cluster rest frame) in its spectrum, the amplitude of the features drops with rising temperature;

5. the interaction of the background with the colder $\left(k T \sim 10^{6} \mathrm{~K}\right)$ plasma located on the distant $(\lesssim 3$ $\mathrm{Mpc})$ periphery of nearby $(z \lesssim 1)$ galaxy clusters noticeably enhances the absorption line at $h \nu \sim 2$ $\mathrm{keV}$ in its spectrum and, moreover, leads to its splitting (the appearance of a broad intense satellite line at $h \nu \sim 1.3 \mathrm{keV}$ whose amplitude does not depend on the properties of the high-temperature cluster gas); the line detection will allow the parameters and distribution of this mysterious medium containing $2 / 3$ of the baryonic matter in the Universe to be investigated;

6. features due to absorption and scattering are also formed in the intrinsic thermal and recombination radiation spectrum of the hot cluster gas; when the thermal spectrum of an optically thin plasma is subtracted from the observed total spectrum, they are perceived as additional background distortions with an amplitude $\sim 100 \%$ or more;

7. the spectral shape of the background distortions depends on the cluster redshift $z$ (in contrast to the spectral shape of the microwave background distortions), although the distortion amplitude does not depend on $z$;

8. the detection of background distortions at energies $h \nu \lesssim 20 k T_{\mathrm{e}}$ is complicated by the presence of intrinsic thermal radiation from the intergalactic gas, which makes it very difficult to measure the effect of a rise in the background in the $\mathrm{X}$-ray range; the observations of the MeV dip in the background spectrum are free from this noise for most clusters;

9. the detection of X-ray background distortions is also complicated by the presence of AGNs in (or near) the cluster with luminosities $L_{\mathrm{X}} \sim 10^{41}-$ $10^{43} \mathrm{erg} \mathrm{s}^{-1}$ at present or even in the distant (up to $\sim 1$ Myr due to the X-ray echo) past, because the $\mathrm{X}$-ray radiation from such galaxies scattered in the cluster becomes diffuse radiation that enhances the background at $h \nu \lesssim 150 \mathrm{keV}$; the scattered radiation does not contain any photoabsorption lines and even the $\mathrm{MeV}$ dip in its spectrum and, therefore, does not change their amplitude in the spectrum of the background distortions.

We considered a number of real galaxy clusters and predicted the shape and amplitude of the spectra of the background deviations expected for them based on the available parameters. Although the background intensity distortions toward the cluster center are maximal for the most massive hot clusters, cold nearby clusters like Virgo and Coma have real chances for detecting the effect in the hard X-ray and gamma-ray range already now. It seems possible that the hard X-ray background fluctuations detected by the HEAO-1 and RXTE observatories are associated in part with the effect being discussed in the paper — with the background distortions in distant galaxy clusters.

\section{CONCLUSIONS}

It is clear from the foregoing that the main obstacle to observing the distortions in the cosmic background spectrum due to scattering by electrons in the X-ray range $h \nu \lesssim 60-100 \mathrm{keV}$ (in the cluster rest frame) is the intrinsic plasma thermal radiation 4 . If it were not for the illumination by this radiation, modern X-ray telescopes with grazing-incidence optics like NuSTAR (Harrison et

\footnotetext{
${ }^{4}$ Besides, although the background distortions due to scattering and photoabsorption grow with increasing $\tau_{\mathrm{T}}$ of the cluster gas, the thermal radiation intensity, proportional to $N_{\mathrm{e}}^{2}$, grows much faster.
} 
al. 2013) might well observe these distortions (or at least they have fallen just short of this). The X-ray calorimeters with the resolution and sensitivity required to measure the absorption line profile in the background being designed at present would be able to determine the gas temperature in a cluster and its composition only from the absorption line profile in the background.

In December 2019 the SRG orbital astrophysical observatory with the highly sensitive ART-XC and eROSITA telescopes onboard is going to begin to scan the sky in X-rays for four years. The ART-XC telescope, operating in the range 5-30 keV (Pavlinsky et al. 2018), will be able to observe the distortions in the background spectrum due to scattering by electrons in the hot gas of clusters and photoabsorption by iron and nickel ions; the eROSITA telescope, operating in the range $0.3-8$ $\mathrm{keV}$ (Predehl et al. 2018), will be able to investigate the absorption lines in the background spectrum at lower energies. Even before the survey, within the Russian part of the Performance Verification Program, the SRG observatory is going to scan the nearby bright Coma cluster.

We could attempt to subtract the thermal radiation spectrum of the cluster gas itself from the measurements using theoretical models and knowing the gas temperature, density, and metallicity. However, (1) in real clusters the gas temperature usually changes with radius and in a rather complicated way, which greatly complicates highly accurate simulations; (2) because of the finite optical depth of the cluster gas, the distortions due to scattering and absorption should appear in the spectrum of its thermal radiation, which are much greater in absolute value than the background distortions that are added to them when subtracting the ideal spectrum of an optically thin plasma; (3) the intense thermal radiation of the gas should introduce a noticeable statistical error into the background measurements, reducing the signal-to-noise ratio $S / N$. Therefore, to detect the effect, it is more preferable to investigate cold, massive, nearby (extended) clusters using the peripheral cluster regions, along with the central ones, for observations.

The presence of AGNs in a galaxy cluster can additionally complicate the X-ray observations of the effect, because their radiation scattered in the cluster gas will be perceived as a "positive" background distortion. Scattered radiation can exist in a cluster even hundreds of thousands of years after the decay of the galactic nucleus - due to the "X-ray echo" effect. At the same time, it contains no absorption lines and $\mathrm{MeV}$ dip in the spectrum and does not distort these features appearing in the X-ray background spectrum when interacting with the hot cluster gas.

It may well be that it would be more preferable to search for the Compton cosmic background distortions in the hard X-ray and gamma-ray spectral ranges, where their relative amplitude is maximal and the contribution of the thermal radiation from the hot cluster gas falls exponentially. However, it should be borne in mind that the absolute amplitude of the distortions in these ranges decreases due to the peculiar shape of the X-ray background spectrum (see Fig.9 9 above). Besides, the instruments operating in these ranges are still noticeably inferior in their capabilities to modern X-ray telescopes, despite the abundance of problems and the general understanding of the importance of studies in this field. The incessant efforts (see, e.g., Fryer et al. 2019) to design more sensitive $\mathrm{MeV}$ gamma-ray telescopes like eASTROGAM (Tatischeff et al. 2016; De Angelis et al. 2017) or ASTENA/LAUE (Virgilli et al. 2017) allow one to look with optimism at the prospects for detecting the effect in this range in the immediate future.

Concluding this paper, we emphasize once again that all of the expected background distortions in the hot cluster gas have a very small $(\lesssim 1 \%$, and as a rule, $\sim 0.1 \%$ ) absolute value. They cannot be seen visually just by examining the measured cluster spectrum. They will manifest themselves only in the relative distortion spectrum, at a very high accuracy of measurements and in proper simulations of the background spectra and the thermal spectrum of the cluster gas, when all of the effects discussed in the paper are taken into account.

\section{ACKNOWLEDGMENTS}

S. A. Grebenev is grateful to R. A. Burenin and S. Yu. Sazonov for the useful discussion and consultations and the Basic Research Program 12 of the Russian Academy of Sciences ("Questions of the Origin and Evolution of the Universe with the Application of Methods of Ground-Based Observations and Space Research") for financial support. R. A. Sunyaev is grateful to the Russian Science Foundation for support by grant no. 19-12-00369. This study was performed within the "Universe" theme of the research program at the Space Research Institute of the Russian Academy of Sciences.

APPENDIX

\section{THE NAVARRO-FRENK-WHITE MODEL}

The dark matter density distribution in galaxy clusters is successfully described by the Navarro-Frenk-White (hereafter NFW) profile (Navarro et al. 1997) found by N-body simulations of equilibrium configurations in the theory of hierarchical clustering of cold dark matter:

$$
\rho_{\mathrm{D}}(R)=\rho_{\mathrm{s}} \frac{R_{\mathrm{s}}}{R}\left(1+\frac{R}{R_{\mathrm{s}}}\right)^{-2} .
$$

Here, $\rho_{\mathrm{s}}$ is the dark matter density parameter and $R_{\mathrm{S}}$ is the scale parameter (cluster core radius). For such 


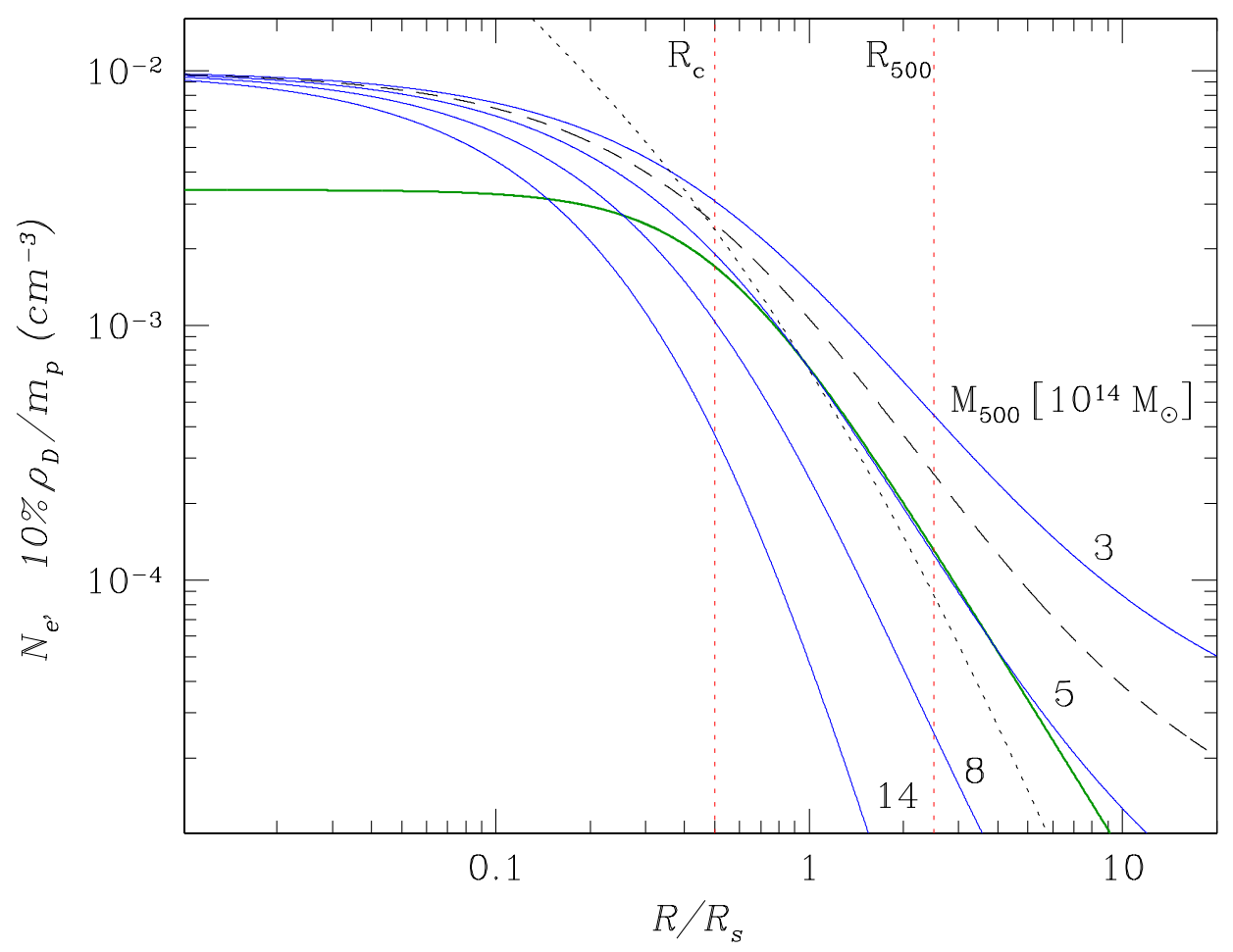

Fig. 14. Equilibrium density distributions of an isothermal gas in the gravitational field of a cluster with dark matter having the NFW profile (Navarro et al. 1997) and their dependence on cluster mass $M_{500}$. The thin solid (blue) lines indicate the distributions with $k T_{\mathrm{e}}=5 \mathrm{keV}$. The central gas density is everywhere assumed to be the same (see the text). The dashed (black) line for $M_{500}=5 \times 10^{14} M_{\odot}$ indicates the distribution with $k T_{\mathrm{e}}=6 \mathrm{keV}$. The concentration parameter is $c=2.5$. The thick (green) line indicates the $\beta$ gas distribution with $\beta=2 / 3$ and $R_{\mathrm{c}}=0.5 R_{\mathrm{s}}$ (the normalization ensures that the $\beta$ distribution near $R \sim R_{\mathrm{s}}$ coincides with the gas distribution in the NFW model for $M_{500}=5 \times 10^{14} M_{\odot}$; the dotted line indicates the $10 \%$ level of the dark matter density distribution $\rho_{\mathrm{D}} / m_{\mathrm{p}}$ for this case).

a density profile the cluster dark matter mass within radius $R$ is

$$
M_{\mathrm{D}}(<R)=4 \pi \rho_{\mathrm{s}} R_{\mathrm{s}}^{3}\left[\ln \left(\frac{R}{R_{\mathrm{s}}}+1\right)-\frac{R}{R+R_{\mathrm{s}}}\right] .
$$

Given the dark matter distribution, the cluster gas distribution law can be found (refined). In particular, in several papers it was proposed to modify the $\beta$ density distribution by including a central cusp and an additional wider component (see, e.g., Vikhlinin et al. 2006; Arnaud et al. 2010). Because of the larger number of parameters, the modified distribution acquired greater freedom in changing the shape and successfully fitted the observed brightness profiles of clusters. A different approach was realized by Shi and Komatsu (2014) and Shi et al. (2016), who used the hydrostatic equilibrium equation for the gas in the gravitational field of dark matter to find the cluster gas distribution. However, they obtained the gas density (pressure) profiles in an explicit form only for specific temperature distributions. As will be shown below, this approach itself can also be extended to the clusters with a constant temperature investigated in this paper.
Neglecting the gas self-gravity, the hydrostatic equilibrium equation has a simple form:

$$
M_{\mathrm{D}}(<R)=-\frac{2 k T_{\mathrm{e}}}{G m_{\mathrm{p}}} \frac{d \ln \left(N_{e}\right)}{d \ln (R)} R,
$$

where $G$ is the gravitational constant and $m_{\mathrm{p}}$ is the proton mass. Substituting (A1) into this equation and integrating the resulting equation, we find the cluster gas density distribution

$$
N_{\mathrm{e}}(R)=N_{\mathrm{s}} e^{-\epsilon}\left(1+\frac{R}{R_{\mathrm{s}}}\right)^{\epsilon R_{\mathrm{s}} / R} .
$$

Here, $N_{\mathrm{s}}$ is the central gas electron density; generally speaking, it is not directly related to the central dark matter density $\rho_{\mathrm{s}}$. The shape of the distribution is defined by only one dimensionless parameter

$$
\epsilon=2 \pi \rho_{\mathrm{s}} R_{\mathrm{s}}^{2}\left(G m_{\mathrm{p}} / k T_{\mathrm{e}}\right) .
$$

Using the characteristic values of the cluster radius $R_{500}$, mass $M_{500}=500 \rho_{\mathrm{cr}}(z)(4 / 3) \pi R_{500}^{3}$, and the concentration parameter $c$ proposed by Navarro et al. (1997) to 
describe the cluster properties:

$$
\begin{aligned}
& R_{500}=c R_{\mathrm{s}} \\
& M_{500}=4 \pi \rho_{\mathrm{s}} R_{\mathrm{s}}^{3} E(c)^{-1}, \text { where } \\
& E(c)=[\ln (1+c)-c /(1+c)]^{-1},
\end{aligned}
$$

and $\rho_{\mathrm{cr}}(z)=3 /(8 \pi G) H(z)^{2}$ is the mean (critical) density of the Universe at cluster redshift $z$, we will transform (A4) to

$$
\begin{gathered}
\epsilon=\frac{G M_{500} m_{\mathrm{p}}}{2 k T_{\mathrm{e}} R_{\mathrm{s}}} E(c) \simeq \\
9.1 c E(c)\left(\frac{M_{500}}{10^{15} M_{\odot}}\right)\left(\frac{R_{500}}{500 \mathrm{kpc}}\right)^{-1}\left(\frac{k T_{\mathrm{e}}}{5 \mathrm{keV}}\right)^{-1} .
\end{gathered}
$$

Figure 14 shows how the equilibrium gas density distributions in a cluster with the NFW dark matter profile calculated from Eqs. A3 and (A4) depend on the cluster mass $M_{500}$ and gas temperature $k T_{\mathrm{e}}$. The concentration parameter $c$ was taken to be 2.5. For the cluster with $M_{500}=5 \times 10^{14} M_{\odot}$ the central gas density $N_{\mathrm{s}}$ was determined by assuming the mass of the gas contained within the radius $R_{500}$ to be $M_{\mathrm{g}}\left(R<R_{500}\right) \simeq 0.1 M_{500}$ (Kravtsov et al. 2006). The central gas density in the remaining clusters was fixed at this value for the convenience of tracing the change in profile shape (obviously, the condition $M_{\mathrm{g}} \simeq 0.1 M_{500}$ no longer must be fulfilled in this case). The gas distribution depends on $k T_{\mathrm{e}}$ as strongly as it does on $M_{500}$ (compare the distribution for the cluster with $M_{500}=5 \times 10^{14} M_{\odot}$ and a gas temperature $k T_{\mathrm{e}}=5 \mathrm{keV}$ indicated by the solid blue line and the distribution for the same cluster, but with $k T_{\mathrm{e}}=6$ $\mathrm{keV}$, indicated by the dashed black line). This would be expected, because these quantities enter into (A5) in the combination $M_{500} / k T_{\mathrm{e}}$.

The thick (green) line in the figure indicates the $\beta$ gas distribution with $\beta=2 / 3$ and $r_{\mathrm{c}}=0.5 R_{\mathrm{s}}$. The characteristic values of $R_{\mathrm{c}}$ and $R_{500}$ are marked in the figure by the vertical dotted (red) lines. The normalization of the gas density was chosen in such a way that this distribution roughly coincided with the gas distribution in the NFW model near $R \sim R_{\mathrm{s}}$. The $\beta$ distribution is seen to have a much gentler (flatter) profile in the central $\left(R \lesssim R_{\mathrm{s}}\right.$ ) part of the cluster than the NFW profile. For comparison, the dotted (black) curve in the figure indicates the NFW dark matter distribution proper, its normalization was set equal to $10 \%$ of the density $\rho_{\mathrm{D}}(R) / m_{\mathrm{p}}$ for $M_{500}=5 \times 10^{14} M_{\odot} 5$. The radial Thomson optical depth for such a cluster computed by integrating the distribution (A3) is $\tau_{\mathrm{r}} \simeq 3.3 \times 10^{-3}$, which accounts for $\sim 2 / 3$ of $\tau_{\mathrm{c}}=\sigma_{\mathrm{T}} N_{\mathrm{s}} R_{\mathrm{c}}$, and is even smaller for more massive clusters. For the $\beta$ distribution with a flat top the radial optical depth is $0.5 \pi \tau_{\mathrm{c}}$ (see Eq.

\footnotetext{
${ }^{5}$ This is how the cluster gas would be distributed if the shape of its radial density profile closely coincided with the shape of the dark matter profile.
}

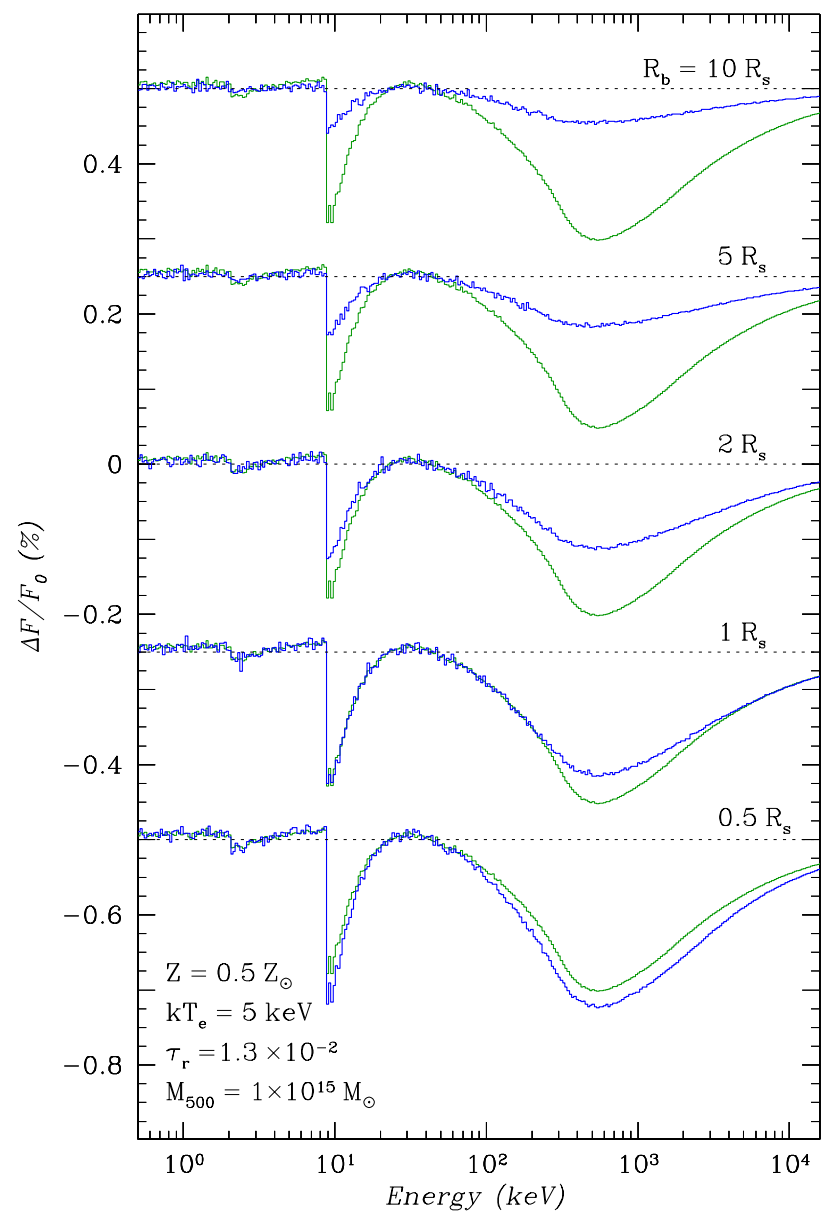

Fig. 15. The relative background distortions (thick blue lines) arising in a cluster where the dark matter is distributed according to the NFW law, while the gas - according to Eq. (A3). The cluster mass is $M_{500}=1 \times 10^{15} M_{\odot}$ (the core radius is $R_{\mathrm{c}} \simeq 300 \mathrm{kpc}$ ), the radial optical depth of the gas is $\tau_{\mathrm{r}} \simeq 1.3 \times 10^{-2}$. The cases with various break radii $R_{\mathrm{b}}$ are considered. The background distortions in a cluster with a uniform density distribution (thin green lines) with a radial optical depth $\tau_{\mathrm{c}}=6 \times 10^{-3}$ are shown for comparison. The gas temperature and metallicity in both cases are the same, $k T_{\mathrm{e}}=5 \mathrm{keV}$ and $Z=0.5 Z_{\odot}$.

(6) ). This difference is related to the more rapid drop in gas density with radius in the central part of the NFW model cluster.

The results of our computations of the cosmic background distortions for a cluster with the NFW dark matter profile are indicated in Fig.15 by the thick (blue) lines. Here we consider a more massive cluster with $M_{500}=1 \times 10^{15} M_{\odot}$, a radial optical depth $\tau_{\mathrm{r}}=$ $1.3 \times 10^{-2}\left(\tau_{\mathrm{c}}=3.8 \times 10^{-2}\right), R_{\mathrm{s}}=600 \mathrm{kpc}\left(R_{\mathrm{c}}=300\right.$ $\mathrm{kpc}$ ), a gas temperature $k T_{\mathrm{e}}=5 \mathrm{keV}$, and metallicity $Z=0.5 Z_{\odot}$. For comparison, the thin (green) lines indicate the results of our computations of the background distortions for a cluster with a uniform gas density with a radial optical depth $\tau_{\mathrm{c}}=\sigma_{\mathrm{T}} N_{\mathrm{e}} R_{\mathrm{c}}=6 \times 10^{-3}$ and the same temperature $k T_{\mathrm{e}}=5 \mathrm{keV}$. We consid- 
ered the cases with various density break radii $R_{\mathrm{b}}=$ $0.5,1,2,5$, and $10 R_{\mathrm{s}}$. Although the real Thomson optical depth of the gas in the NFW cluster exceeds that in the homogeneous model by a factor of $\sim 2$, the distortions arising in the NFW model due to the strong gas concentration to the center are comparable to the distortions in a cluster with a uniform density (for the computations with $R_{\mathrm{b}} \leq 1 R_{\mathrm{s}}$ ). For larger $R_{\mathrm{b}}$ the distortion amplitude drops in the same way as in the case of a cluster with a $\beta$ density distribution (Fig.5). Obviously, the distortion depth is determined by the optical depth averaged over the visible part of the cluster $\left\langle\tau_{\mathrm{T}}\right\rangle$. Just as in the case of a $\beta$ distribution, it is clear that the efficiency of background distortion observations by a telescope with an aperture (angular resolution) with a radius more than $\sim 1-2 R_{\mathrm{c}}$ (at given $z$ ) should drop rapidly.

\section{REFERENCES}

1. P. A. R. Ade, N. Aghanim, M. Arnaud, M. Ashdown, F. Atrio-Barandela, J. Aumont, C. Baccigalupi, A. Balbi, et al. (Planck Collaboration), Astron. Astrophys. 554, A140 (2013)

2. P. A. R. Ade, N. Aghanim, C. Armitage-Caplan, M. Arnaud, M. Ashdown, F. Atrio-Barandela, J. Aumont, H. Aussel, et al. (Planck Collaboration), Astron. Astrophys. 571, A29 (2014).

3. P. A. R. Ade, N. Aghanim, C. Armitage-Caplan, M. Arnaud, M. Ashdown, F. Atrio-Barandela, J. Aumont, H. Aussel, et al. (Planck Collaboration), Astron. Astrophys. 581, A14 (2015).

4. P. A. R. Ade, N. Aghanim, M. Arnaud, M. Ashdown, J. Aumont, C. Baccigalupi, A. J. Banday, R.B. Barreiro, et al. (Planck Collaboration), Astron. Astrophys. 594, A27 (2016a).

5. P. A. R. Ade, N. Aghanim, M. Arnaud, M. Ashdown, J. Aumont, C. Baccigalupi, A. J. Banday, R.B. Barreiro, et al. (Planck Collaboration), Astron. Astrophys. 596, A101 (2016b).

6. N. Aghanim, M. Arnaud, M. Ashdown, F. AtrioBarandela, J. Aumont, C. Baccigalupi, A. Balbi, A. J. Banday, et al. (Planck Collaboration), Astron. Astrophys. 536, A26 (2011).

7. C.W. Allen, Astrophysical Quantities, 3d ed. (Athlone, London, 1973).

8. A. de Angelis, V. Tatischeff, M. Tavani, U. Oberlack, I. Grenier, L. Hanlon, R. Walter, A. Argan, P. von Ballmoos, et al., Exp. Astron. 44, 25 (2017).

9. M. Arnaud, Astron. Astrophys. 500, 103 (2009).

10. M. Arnaud, G. W. Pratt, R. Piffaretti, H. Böhringer, J. H. Croston, and E. Pointecouteau, Astron. Astrophys. 517, A92 (2010).

11. J. Arons, Astrophys. J. 164, 437 (1971).

12. M. Birkinshaw, Phys. Rep. 310, 97 (1999).

13. L. E. Bleem, B. Stalder, T. de Haan, K. A. Aird, S. W. Allen, D. E. Applegate, M. L. N. Ashby, M. Bautz, et al., Astrophys. J. Suppl. Ser. 216, 27 (2015).
14. E. Boldt, Phys. Rep. 146, 215 (1987).

15. S. Boughn and R. Crittenden, Nature 427, 45 (2004).

16. S. P. Boughn and R. G. Crittenden, Mon. Not. Roy. Astron. Soc. 360, 1013 (2005).

17. E. Bulbul, I.-N. Chiu, J. J. Mohr, M. McDonald, B. Benson, M. W. Bautz, M. Bayliss, and L. Bleem, Astrophys. J. 871, 50 (2019).

18. J. E. Carlstrom, G. P. Reese, and D. Erik, Ann. Rev. Astron. Astrophys. 40, 643 (2002).

19. A. Cavaliere and R. Fusco-Femiano, Astron. Astrophys. 49, 137 (1976).

20. R. Cen and J. P. Ostriker, Astrophys. J. 514, 1 (1999).

21. E. Churazov, M. Haehnelt, O. Kotov, and R. Sunyaev, Mon. Not. Roy. Astron. Soc. 323, 93 (2001).

22. E. Churazov, R. Sunyaev, M. Revnivtsev, S. Sazonov, S. Molkov, S. Grebenev, C. Winkler, A. Parmar, et al., Astron. Astrophys. 467, 529 (2007).

23. G. Cooper, Phys. Rev. D 3, 2312 (1971).

24. L. P. David, A. Slyz, C. Jones, W. Forman, S. D. Vrtilek, and K. A. Arnaud, Astrophys. J. 412, 479 (1993).

25. A. C. Fabian, Nature 269, 672 (1977).

26. A. Finoguenov, U.G. Briel, and J.P. Henry, Astron. Astrophys. 410, 777 (2003).

27. W. Forman and C. Jones, Ann. Rev. Astron. Astrophys. 276, 547 (1982).

28. C. L. Fryer, F. Timmes, A.L. Hungerford, A. Couture, F. Adams, M. Avila, W. A. Aoki, A. Arcones, D. Arnett, et al., "A White Paper for the 2020 Decadal Survey", astro-ph:1902.02915 (2019).

29. M. Fukugita, C. Hogan, and P. J. E. Peebles, Astrophys. J. 503, 518 (1998).

30. R. Gavazzi , C. Adami, F. Durret, J.-C. Cuillandre, O. Ilbert, A. Mazure, R. Pelló, and M. P. Ulmer, Astron. Astrophys. 498, L33 (2009).

31. R. Giacconi, P. Rosati, P. Tozzi, M. Nonino, G. Hasinger, C. Norman, J. Bergeron, S. Borgani, et al., Astrophys. J. 551, 624 (2001).

32. S. A. Grebenev and R. A. Sunyaev, Sov. Astron. Lett. 13, 438 (1987).

33. S. A. Grebenev and R. A. Sunyaev, Mon. Not. Roy. Astron. Soc., in preparation (2020).

34. D. E. Gruber, J. L. Matteson, L. E. Peterson, and G. V. Jung, Astrophys. J. 520, 124 (1999a).

35. D. E. Gruber, D. MacDonald, R. E. Rothschild, E. Boldt, R. F. Mushotzky, and A. C. Fabian, Nucl. Phys. B - Proc. Sup. 69, 625 (1999b).

36. F. A. Harrison, W. W. Craig, F. E. Christensen, C. J. Hailey, W. W. Zhang, S. E. Boggs, D. Stern, W. R. Cook, et al., Astrophys. J. 770, 103 (2013).

37. G. Hasinger, R. Burg, R. Giacconi, M. Schmidt, J. Trumper, and G. Zamorani, Astron. Astrophys. 329, 482 (1998).

38. M. Hasselfield, M. Hilton, T. A. Marriage, G. E. Addison, L. F. Barrientos, N. Battaglia, E. S. Battistelli, J.R. Bond, et al., J. Cosmol. Astropart. Phys. 07, 008 (2013). 
39. T. Herbig, C. R. Lawrence, A. C. S. Readhead, and S. Gulkis, Astrophys. J. 449, L5 (1995).

40. A. F. Illarionov and R. A. Syunyaev, Sov. Astron. 16, 45 (1972).

41. C. Jones and W. Forman, Astrophys. J. 276, 38 (1984).

42. J. S. Kaastra, Proc. IAU Colloquium "Outskirts of Galaxy Clusters: Intense Life in the Suburbs" (ed. A. Diaferio) 195, 105 (2004a).

43. J. S. Kaastra, J. Korean Astron. Soc. 37, 375 (2004b).

44. R. Khatri and R. A. Sunyaev, in preparation (2019).

45. A. S. Kompaneets, Sov. Phys. JETP 4, 730 (1957).

46. A. V. Kravtsov, A. Vikhlinin, and D. Nagai, Astrophys. J. 650, 128 (2006).

47. M. S. Longair and R. A. Sunyaev, Nature 223, 719 (1969).

48. D. R. MacDonald, D. E. Gruber, and E. A. Boldt, AIP Conf. Proc 599, 734 (2001).

49. P. Madau and G. Efstathiou, Astrophys. J. 517, L9 (1999).

50. M. Markevitch, G. R. Blumenthal, W. Forman, C. Jones, and R. A. Sunyaev, Astrophys. J. 395, 326 (1992).

51. M. Markevitch, R. A. Sunyaev, and M. N. Pavlinsky, Nature 364, 40 (1993).

52. M. Markevitch, A. H. Gonzalez, L. David, A. Vikhlinin, S. Murray, W. Forman, C. Jones, and W. Tucker, Astrophys. J. 567, L27 (2002).

53. M. McDonald, B. R. McNamara, R. J. van Weeren, D. E. Applegate, M. Bayliss, M. W. Bautz, B. A. Benson, J. E. Carlstrom, et al., Astrophys. J. 811, 111 (2015).

54. F. Menanteau, J. P. Hughes, C. Sifón, M. Hilton, J. González, L. Infante, L. F. Barrientos, A. J. Baker, et al., Astrophys. J. 748, 7 (2012).

55. T. Miyaji, G. Hasinger, and M. Schmidt, Astron. Astrophys. 353, 25 (2000).

56. T. Miyaji, G. Hasinger, M. Salvato, M. Brusa, N. Cappelluti, F. Civano, S. Puccetti, M. Elvis, et al., Astrophys. J. 804, 104 (2015).

57. J. F. Navarro, C. S. Frenk, and S. D. M. White, Astrophys. J. 490, 493 (1997).

58. M. Pavlinsky, V. Levin, V. Akimov, A. Krivchenko, A. Rotin, M. Kuznetsova, I. Lapshov, A. Tkachenko, et al., Proc. SPIE 10699, 106991Y (2018).

59. L. A. Pozdnyakov, I. M. Sobol', and R. A. Syunyaev, Sov. Sci. Rev., Sec. E: Astrophys. Space Phys. Rev. 2, 189 (1983).

60. P. Predehl, W. Bornemann, H. Bräuninger, H. Brunner, V. Burwitz, D. Coutinho, K. Dennerl, J. Eder, et al., Proc. SPIE 10699, 106995H (2018).

61. M. Rauch Ann. Rev. Astron. Astrophys. 36, 267 (1998).

62. J. C. Raymond and B. W. Smith, Astrophys. J. Suppl. Ser. 35, 419 (1977).

63. S. Yu. Sazonov and R. A. Sunyaev, Astrophys. J. 543, 28 (2000).

64. S. Yu. Sazonov, R. A. Sunyaev, and C. K. Cramphorn, Astron. Astrophys. 393, 793 (2002).
65. S. Sazonov, R. Krivonos, M. Revnivtsev, E. Churazov, and R. Sunyaev, Astron. Astrophys. 482, 517 (2008).

66. S. L. Shapiro, A. P. Lightman, and D. M. Eardley, Astrophys. J. 204, 187 (1976).

67. X. Shi and E. Komatsu, Mon. Not. Roy. Astron. Soc. 442, 521 (2014).

68. X. Shi, E. Komatsu, D. Nagai, and E. T. Lau, Mon. Not. Roy. Astron. Soc. 455, 2936 (2016).

69. R. A. Sunyaev, Sov. Astron. Lett. 6, 213 (1980).

70. R. A. Sunyaev, M. Markevitch, and M. Pavlinsky, Astrophys. J. 407, 606 (1993).

71. R. A. Sunyaev and L. G. Titarchuk, Astron. Astrophys. 86, 121 (1980).

72. R. A. Sunyaev and Ya. B. Zeldovich, Ap\&SS 7, 3 (1970).

73. R. A. Sunyaev and Ya. B. Zeldovich, Comm. Astrophys. Space Phys. 4, 173 (1972).

74. R. A. Sunyaev and Ya. B. Zeldovich, Ann. Rev. Astron. Astrophys. 18, 537 (1980).

75. R. A. Sunyaev and Ya. B. Zeldovich, Sov. Sci. Rev., Sec. E: Astrophys. Space Phys. Rev. 1, 1 (1981).

76. V. Tatischeff, M. Tavani, P. von Ballmoos, L. Hanlon, U. Oberlack, A. Aboudan, A. Argan, D. Bernard, et al., Proc. SPIE 9905, 99052N (2016).

77. M. Treyer, C. Scharf, O. Lahav, K. Jahoda, E. Boldt, and T. Piran, Astrophys. J. 509, 531 (1998).

78. Y. Ueda, M. Akiyama, G. Hasinger, T. Miyaji, and M. G. Watson, Astrophys. J. 786, 104 (2014).

79. L. A. Vainshtein and R. A. Sunyaev, Sov. Astron. Lett. 6, 353 (1980).

80. D. A. Verner and D. G. Yakovlev, Astron. Astrophys. Suppl. Ser. 109, 125 (1995).

81. D. A. Verner, G. J. Ferland, K. T. Korista, and D. G. Yakovlev, Astrophys. J. 465, 487 (1996).

82. A. Vikhlinin, A. Kravtsov, W. Forman, C. Jones, M. Markevitch, S. S. Murray, and L. van Speybroeck, Astrophys. J. 640, 691 (2006).

83. E. Virgilli, V. Valsan, F. Frontera, E. Caroli, V. Liccardo, and J. B. Stephen, JATIS 3(4), 044001 (2017); arXiv:1711.03475.

84. D. A. White, C. Jones, and W. Forman, Mon. Not. Roy. Astron. Soc. 292, 419 (1997).

85. R. Williamson, B. A. Benson, F. W. High, K. Vanderlinde, P. A. R. Ade, K. A. Aird, K. Andersson, R. Armstrong, et al., Astrophys. J. 738, 139 (2011).

86. Ya. B. Zel'dovich and I. D. Novikov, The Structure and Evolution of the Universe (M.: Nauka, 1975).

87. Ya. B. Zel'dovich and R. A. Sunyaev, in Astrophysics and Space Physics (ed. by R. A. Sunyaev, Nauka-Fizmatlit, Moscow, 1982), p. 9 [in Russian].

Translated by $V$. Astakhov 